hep-th/0507284

CERN-PH-TH/2005-134

July 2005

\title{
The algebraic structure of geometric flows in two dimensions
}

\author{
Ioannis Bakas* \\ Theory Division, Department of Physics, CERN \\ CH-1211 Geneva 23, Switzerland \\ ioannis.bakas@cern.ch
}

\begin{abstract}
There is a common description of different intrinsic geometric flows in two dimensions using Toda field equations associated to continual Lie algebras that incorporate the deformation variable $t$ into their system. The Ricci flow admits zero curvature formulation in terms of an infinite dimensional algebra with Cartan operator $\partial / \partial t$. Likewise, the Calabi flow arises as Toda field equation associated to a supercontinual algebra with odd Cartan operator $\partial / \partial \theta-\theta \partial / \partial t$. Thus, taking the square root of the Cartan operator allows to connect the two distinct classes of geometric deformations of second and fourth order, respectively. The algebra is also used to construct formal solutions of the Calabi flow in terms of free fields by Bäcklund transformations, as for the Ricci flow. Some applications of the present framework to the general class of Robinson-Trautman metrics that describe spherical gravitational radiation in vacuum in four space-time dimensions are also discussed. Further iteration of the algorithm allows to construct an infinite hierarchy of higher order geometric flows, which are integrable in two dimensions and they admit immediate generalization to Kähler manifolds in all dimensions. These flows provide examples of more general deformations introduced by Calabi that preserve the Kähler class and minimize the quadratic curvature functional for extremal metrics.
\end{abstract}

\footnotetext{
* On sabbatical leave from Department of Physics, University of Patras, GR-26500 Patras, Greece; e-mail: bakas@ajax.physics.upatras.gr
} 


\section{Introduction}

The subject of geometric flows witnessed rapid development in recent years. It only fair to say that many areas of mathematics underwent rapid development as new tools became available for addressing long standing open problems - predominantly in differential geometry - via appropriately chosen evolution equations, also known as geometric flows. At the same time, several cases of such flows arose independently in physics with applications ranging from classical mechanics to general relativity and quantum field theory. Roughly speaking, there are two general classes of deformation equations in differential geometry, namely intrinsic and extrinsic curvature flows. The former refer to geometric evolutions of Riemannian metrics $g$ on a given manifold $M$ that are driven by the Ricci curvature, in various forms, whereas the latter refer to geometric evolutions of submanifolds embedded in $M$ that are driven by their extrinsic curvature. As such, they all correspond to dynamical systems in superspace, which is the infinite dimensional space of all possible metrics on a given manifold. The geometric flows provide systems of parabolic equations, being first order in the deformation variable $t$ that is typically called time, and they are second or higher order in the space variables. However, the equations are quite difficult to solve in all generality, due to non-linearities. Although the short time existence of solutions for a given initial metric is guaranteed by the parabolic nature of the equations, their convergence to canonical metrics after sufficiently long time has only been analyzed under various conditions, and in connection to the problem of formation of singularities along the flows.

All geometric flows share some common qualitative features with the linear heat flow equation, which has the tendency to dissipate any temperature perturbations all over space after sufficiently long time. Thus, curvature perturbations around canonical metrics tend to wash away after infinitely long time, provided that no singularities are formed along the way and that the corresponding geometric deformations are taken in some normalized form, so that the volume of the space remains invariant in time. It is precisely for this reason that continuous flows to constant curvature metrics have been particularly successful for exploring the relations among the geometric and topological structures of manifolds and prove various geometrization conjectures in low dimensions, as in the classic uniformization problem of Riemann surfaces. However, there is no general dictionary at this moment that connects geometric evolution equations to physical problems, apart from some special examples that are under intense investigation in recent years. It is quite plausible that field theory, classical as well as quantum, may provide a unifying framework for all different species of geometric flows by giving appropriate

physical interpretation to the deformation variable $t$ and to the structure of their driving terms.

The Ricci flow is the prime example of this kind as it describes the beta function equations of two dimensional sigma models to lowest order in perturbation theory. The Calabi flow, on the other hand, makes its appearance in general relativity while studying the general class of four-dimensional Robinson-Trautman radiative metrics. Both these 
flows correspond to intrinsic geometric deformations and they will occupy most of the present work. In fact, it proves advantageous to treat both of them in parallel, as we uncover some formal relations among the two, which exist in two dimensions and generalize quite naturally to Kähler manifolds in all dimensions. Thus, some known techniques for the integration of Ricci flows will be found to admit immediate generalization to the Calabi flow, using the appropriate mathematical framework that will be developed in due course. As byproduct, the formal construction of solutions describing spherical gravitational waves in vacuum will be achieved in the context of four-dimensional general relativity, thus providing the main physical application of this work. Although our analysis is only applicable, as it stands, to intrinsic geometric flows, it is worth noting in passing that other important classes of evolution equations, such as those correspond to extrinsic curvature flows, might become tractable by similar methods. Such generalizations are mostly interesting for a variety of problems in boundary quantum field theory, but they will not be included here.

The primary aim is to develop some new algebraic techniques for casting intrinsic geometric flows in two dimensions into zero curvature form and prove their integrability, in a certain sense, using infinite dimensional Lie algebras. These results should be considered complementary to the traditional techniques used in the mathematics literature for studying general properties and qualitative features of geometric evolution equations. Although strictly limited to two dimensions, where most of the algebraic techniques are becoming available, the results might also be of more general value given the embedding of two-dimensional manifolds into higher dimensional spaces that deform geometrically in one way or another. The present description views the geometric flows as Toda field equations associated to infinite dimensional algebras that incorporate the deformation variable $t$ into the system of their defining commutation relations. This approach works well for the two-dimensional Ricci flow, but it also generalizes quite naturally to the Calabi flow using a formal relation between the two via super-evolution. In fact, as will be seen later, one follows from the other by taking the square root of the time evolution operator, and, thus, their formal integration by group theoretical methods appears to have many similarities. This relation will be further generalized to induce an infinite hierarchy of higher order evolution equations and associated infinite dimensional Lie algebras that enable to cast them in zero curvature form.

The algebras used for the purposes of the present work deserve further study as they fall in the general (but poorly understood) class of infinite dimensional Lie algebras with infinite growth. Two-dimensional integrable systems based on infinite dimensional Lie algebras are quite interesting to study on their own, in all generality, and in comparison to the better understood cases of integrable systems that admit zero curvature formulation with finite dimensional gauge algebras. A key point in our analysis is the uneven treatment of the space coordinates and the time $t$, which is fully encoded into the structure of the infinite dimensional algebra used for the effective two-dimensional description of the equations. Thus, the systems appear to be integrable in the two-dimensional sense, whereas at the same time exhibit dissipative behavior in time without reaching a contra- 
diction of terms. Finally, the algebraic structures underlying these flows might be of more general value if could be extended to encompass other interesting dynamical phenomena in geometrical theories with relevance to the physical world, such as gravitation.

Actually, the framework that is developed here, based on Toda field equations for infinite dimensional algebras, admits a smooth limit when $t$-independent solutions of the two-dimensional Calabi flow are investigated. This class of solutions is also of paramount importance in general relativity as they describe Robinson-Trautman space-times of a certain algebraic type. In this case, as will be seen in detail, there is a zero curvature formulation of the equation using Kac's $K_{2}$ simple Lie algebra with infinite growth. The results that we derive will also be compared to other works on this subject and they will be further used to construct formal power series solutions in terms of free fields as in ordinary Toda systems. As byproduct, a new description of the $K_{2}$ algebra will be obtained within the general framework of supercontinual Lie algebras that are introduced here for the first time and used for the zero curvature formulation of the Calabi flow, its variants, and descendants. Thus, the systematic study and classification of such novel algebraic structures seems to be a valuable problem of common interest in physics and mathematics.

Some generalizations to geometric flows on Kähler manifolds of arbitrary dimension will also be considered. In that general context, the hierarchy of geometric flows provide special examples of a more general deformation problem posed by Calabi for minimizing the quadratic curvature functional, while preserving the Kähler class of the metrics. The standard Calabi flow is the most elementary example in this class of deformations with known physical applications. All of them, however, are naturally associated to Calabi's variational problem in the space of all possible metrics on a given Kähler manifold with Euler-Lagrange equations satisfied by extremal metrics, such as metrics with constant curvature. Thus, they have equal importance in geometry.

The structure of this paper is organized as follows. In section 2, after briefly reviewing the general set up of the main equations, the emphasis is subsequently placed on deformations of two-dimensional geometries, where the new algebraic techniques are becoming available for their (formal) integration by group theoretical methods. In section 3, we give account of the notion of continual Lie algebras, their main properties and the systems of Toda field equations associated to them in zero curvature form. The general method of integration is also illustrated for general choices of Cartan operators and further generalizations to the class of supercontinual Lie algebras are introduced. In section 4, applications of the algebraic method to the two-dimensional Ricci flow are briefly summarized together with the formal construction of the general solution in terms of free fields by Bäcklund transformation. In section 5, the method is extended to the two-dimensional Calabi flow by introducing an infinite dimensional algebra whose structure is dictated by the super-evolution operator that connects it to the Ricci flow. In this context, it is more natural to view the zero curvature formulation of the Calabi flow as providing two-dimensional integrable system for a supercontinual Lie algebra, where the generators depend on the coordinates $(t, \theta)$ of $R^{1 \mid 1}$ superspace. This reformulation 
also proves useful for the description of the general solution in terms of free fields, as for the Ricci flow. In section 6, the relation of the two-dimensional Calabi flow to the theory of spherical gravitational waves in vacuum is spelled out in detail using the class of Robinson-Trautman metrics that represent gravitational radiation in the exterior of bounded sources in four space-time dimensions. Then, genuine solutions of the Calabi flow correspond to type II gravitational backgrounds in Petrov's classification of spacetime geometries. Type III metrics, which correspond to time independent solutions of the Calabi flow, are studied separately in section 7 by Toda field theory techniques and the connections to Kac's $K_{2}$ algebra are spelled out in great detail. In section 8 , an infinite hierarchy of higher order geometric flows is constructed in two dimensions by iterating the formal relation between the Ricci and Calabi flows, via super-evolutions, and their zero curvature formulation is studied in all generality. In section 9, this hierarchy is shown to admit immediate generalization to Kähler manifolds in all dimensions and the resulting evolution equations provide special examples of a more general problem posed by Calabi for deforming arbitrary Kähler metrics toward extremal metrics. Finally, section 10 contains our conclusions and a brief outline of some directions for further work.

\section{Intrinsic geometric evolutions}

In this section we present an account of the Ricci and Calabi flows, as they are defined in Kähler geometry, and then reveal a formal relation between the two that plays important role later. Although our results are mostly confined to one complex (or two real) dimensions, a more general, but brief, introduction is necessary in order to understand which of them can be extended to arbitrary dimensions.

\subsection{Preliminaries}

Let $M$ denote a complex $n$-dimensional manifold, which for all practical purposes is taken to be connected and compact without boundary. Assume also that $M$ admits a Kähler metric $g$, which is locally expressible in the form

$$
d s^{2}=2 g_{a \bar{b}} d z^{a} d \bar{z}^{b}
$$

using a system of holomorphic coordinates $z^{a}$ and their complex conjugates $\bar{z}^{a}$ with $a=1,2, \cdots, n$. The Kähler condition on the metric is expressed by the existence of a locally defined, real valued, smooth function $K(z, \bar{z})$, which is not unique, such that $g_{a \bar{b}}(z, \bar{z})=\partial_{a} \bar{\partial}_{b} K(z, \bar{z})$. We also consider the associated real valued exterior form of degree $(1,1)$,

$$
\omega=\sqrt{-1} g_{a \bar{b}} d z^{a} \wedge d \bar{z}^{b}
$$

which is closed and determines the principal cohomology class of the metric, $[\omega]$. As such, it is an intrinsic invariant of the complex analytic structure on $M$. We are interested 
only in the space of Kähler metric in a fixed cohomology class; other Kähler metrics of the same class can be obtained by the transformation

$$
\tilde{g}_{a \bar{b}}=g_{a \bar{b}}+\frac{\partial^{2} u}{\partial z^{a} \partial \bar{z}^{b}},
$$

where $u$ is a globally defined real valued function on $M$.

For later use it is convenient to recall the expression for the Ricci curvature tensor, in complex notation,

$$
R_{a \bar{b}}=-\frac{\partial^{2} \log (\operatorname{det} g)}{\partial z^{a} \partial \bar{z}^{b}}
$$

and the Ricci scalar curvature, which is

$$
R=\frac{1}{2} g^{\mu \nu} R_{\mu \nu}=g^{a \bar{b}} R_{a \bar{b}} .
$$

Note that the normalization used here is one half of the usual value found in most texts. Finally, the volume element is

$$
d V(g)=\omega^{[n]}=(\sqrt{-1})^{n} \operatorname{det}\left(g_{a \bar{b}}\right) d z^{1} \wedge d \bar{z}^{1} \wedge \cdots \wedge d z^{n} \wedge d \bar{z}^{n}
$$

and it is also an invariant of the complex analytic structure on $M$.

The Laplace-Beltrami operator for a Kähler metric $g$ on $M$ is defined as usual, using the analyst's sign,

$$
\Delta \varphi=\frac{1}{2} g^{i j} \nabla_{i} \nabla_{j} \varphi=g^{a \bar{b}} \nabla_{a} \bar{\nabla}_{b} \varphi=g^{a \bar{b}} \frac{\partial^{2} \varphi}{\partial z^{a} \partial \bar{z}^{b}} .
$$

The integral $\int \Delta \varphi d V(g)$ over the entire space vanishes by Stoke's theorem, since it has been assumed that $M$ is compact without boundaries. Also, for later use, it is convenient to recall the definition of the fourth order operator, $D_{L}$, which is an elliptic differential operator that acts on scalar functions as follows,

$$
\begin{aligned}
D_{L} \varphi & =g^{a \bar{n}} \frac{\partial}{\partial z^{a}}\left(g^{b \bar{c}} \frac{\partial}{\partial z^{b}}\left(g_{l \bar{n}} \frac{\partial}{\partial \bar{z}^{c}}\left(g^{l \bar{m}} \frac{\partial \varphi}{\partial \bar{z}^{m}}\right)\right)\right) \\
& =\Delta \Delta \varphi+\varphi^{a \bar{b}} R_{a \bar{b}}+\varphi^{, a} R_{, a} .
\end{aligned}
$$

It is a strongly elliptic operator that coincides with its complex conjugate $\bar{D}_{L}$ if and only if $R$ is constant and, furthermore, satisfies the relation $D_{L} R=\bar{D}_{L} R$ for any Kähler metric.

The geometric flows that will be considered next provide deformations of the Kähler metrics within a given cohomology class $[\omega]$. As such they can be formulated as parabolic evolution equations for the single function $u(z, \bar{z} ; t)$ that arises in the class of transformations (2.3), or equivalently $\tilde{\omega}=\omega+\partial \bar{\partial} u$. However, we will follow the standard presentation based on evolution equations for the components of the metric itself, which are driven by the intrinsic curvature of $M$ in various forms, depending on the type of flow, and which characterize the order of the non-linear differential equations. 
In all cases, the metric is prescribed at some initial time, say $t=0$, and evolves accordingly to later times. Short time solutions always exist, due to the parabolic nature of the equations, but their long time existence is not guaranteed and depends on various conditions and on the dimensionality of the manifold $M$. When they exist, however, have the tendency to converge to canonical metrics defined in appropriate ways. The advantage of using Kähler geometry is that all information about the curvature is encoded into a single function, the scalar curvature $R$, as in two dimensions. However, many important details also depend on the dimensionality of the Kähler manifold and its properties.

\subsection{Ricci flow}

The Ricci flow is the simplest example of a geometric deformation, which is driven by the Ricci curvature tensor associated to a metric $g$ on the manifold $M$ and assumes the following form (see, for instance, $[1,2]$, and references therein)

$$
\partial_{t} g_{a \bar{b}}=-R_{a \bar{b}}
$$

Actually, this equation can be defined on any Riemannian manifold of arbitrary dimension, but we only consider here its application to Kähler geometry. More general, the Ricci flow takes the form

$$
\partial_{t} g_{\mu \nu}=-R_{\mu \nu}+\nabla_{\mu} \xi_{\nu}+\nabla_{\nu} \xi_{\mu}
$$

thus also taking into account the effect of arbitrary (time dependent) reparametrizations associated to a vector field $\xi$. It is a parabolic second order non-linear differential equation that arose independently in physics and mathematics for different reasons.

In physics it has definite meaning within the renormalization group analysis of twodimensional sigma models, as it provides the rate of change of their target space metric viewed as generalized coupling - with respect to the logarithm of the world-sheet length scale, $[3,4]$. The Ricci curvature tensor arises as the beta function of the target space metric, to lowest order in perturbation theory of the quantum theory, and drives the evolution of the generalized coupling from the ultra-violet to the infra-red regime. Solutions to this equation do not correspond to conformal field theories, but they rather describe transitions between different closed string vacua in the weak gravitational regime of the theory induced by tachyon condensation. In this context, reparametrizations in target space that are associated to gradient vector fields, $\xi_{\mu}=\nabla_{\mu} \phi$, can be interpreted as having a dilaton field $\phi$ in target space coupled to the metric. Likewise, fixed points of the generalized Ricci flow equation (2.10), satisfying the Ricci soliton equation $R_{\mu \nu}=2 \nabla_{\mu} \nabla_{\nu} \phi$, correspond to non-trivial conformal field theories. The Euclidean black-hole in two-dimensional target space with the shape of a semi-infinite long cigar provides the simplest example of such a Ricci-Kähler soliton with axial symmetry.

Another interesting application arises from the possibility to embed the Ricci flow into Einstein equations using two extra dimensions with coordinates $u$ and $v$, as follows,

$$
d s^{2}=-2 d u d v+g_{\mu \nu}(x ; u) d x^{\mu} d x^{\nu},
$$


provided that the light-cone coordinate $u$ is identified with $t$ and there is also a dilaton field linear in $v$ coupled to the higher dimensional metric, [5]. This prescription allows to construct gravitational backgrounds - though of special type - for consistent string propagation, using solutions of the Ricci flow equation in two dimensions lower. Then, the renormalization group flow becomes the profile of a gravitational wave in space-time, and the geometry interpolates between fixed points of the flow in transverse space as one traverses the wave. In effect, this construction makes the Ricci flow to appear as special case of Einstein equations, with the appropriate ansatz, and it is the closest one can get to a similar derivation of the (two-dimensional) Calabi flow from Einstein equations, as will be seen later.

In mathematics, it is often appropriate to use the normalized Ricci flow, which is defined as follows, [1, 2],

$$
\partial_{t} g_{\mu \nu}=-R_{\mu \nu}+\frac{<R>}{\operatorname{dim} M} g_{\mu \nu}
$$

where $\langle R\rangle$ is the average (mean) scalar curvature

$$
<R>=\frac{\int_{M} R[g] d V(g)}{\int_{M} d V(g)},
$$

which is independent of the coordinates on the manifold and can only depend on $t$. The compensating term is designed so that the volume of the space $M$ is preserved under continuous deformations, unlike the standard (unnormalized) Ricci flow that changes it in time. Thus, the normalized flow is more useful as tool for proving various uniformization theorems in geometry, since its solutions do not become extinct after some finite time, but they have better chance to exist for sufficiently long time and converge to canonical (constant curvature) metrics that correspond to the fixed points of equation (2.12) on $M$. However, the two variants of the flow are related to each other by performing rescaling of the metric and time reparametrization according to the rule

$$
\tilde{g}_{\mu \nu}=f(t) g_{\mu \nu}, \quad \tilde{t}=\int d t f(t)
$$

which sets $<\tilde{R}>(\tilde{t})=(\operatorname{dim} M) f^{\prime}(t) / f^{2}(t)$.

In Kähler geometry, in particular, the Ricci flow has been employed for studying the existence of Kähler-Einstein metric on manifolds under appropriate technical conditions. It has already been applied successfully to two dimensional Riemann surfaces of genus 0, 1 or $g \geq 2$ by re-deriving the classic uniformization theorem of Poincaré that asserts the existence of constant curvature metrics with values $+1,0$ or -1 , respectively; as a result, these surfaces are quotients of $S^{2}, R^{2}$ or $H^{2}$ by a discrete subgroup $\Gamma$ acting freely and isometrically. In higher dimensions the situation is promising but more complex. 


\subsection{Calabi flow}

The Calabi flow, on the other hand, which is only defined for Kähler manifolds, assumes the following form, $[6,7]$,

$$
\partial_{t} g_{a \bar{b}}=\frac{\partial^{2} R}{\partial z^{a} \partial \bar{z}^{b}}
$$

It is volume preserving deformation without need for adjustment, unlike the Ricci flow, and it also preserves the Kähler class of the metric. The Calabi flow is a parabolic equation for the components of the metric, or equivalently for the Kähler potential, but it is fourth order in the variables $z$ and $\bar{z}$ and, thus, more complicated to analyze in general. Critical points of the flow are called extremal metrics and they clearly encompass constant curvature metrics, if they exist on a given Kähler manifold. In this respect, the Calabi flow is used as a tool for studying the conditions for Einstein-Kähler metrics in geometry, and in conjunction with their possible obstructions.

There is a variational problem in the space of Kähler metrics that allows to formulate the problem in general terms. In particular, consider the quadratic curvature functional

$$
S(g)=\int_{M} R^{2}[g] d V(g)
$$

defined for all possible metrics on $M$ with fixed cohomology class [ $\omega]$. By computing the variation of $S(g)$ with respect to the flow (2.15) one finds

$$
\partial_{t} S(g)=\int_{M}\left(-2 R \Delta \Delta R-2 R R^{a \bar{b}} R_{a \bar{b}}+R^{2} \Delta R\right) d V(g),
$$

since the associated scalar curvature flows as

$$
\partial_{t} R=-\Delta \Delta R-R^{a \bar{b}} R_{a \bar{b}}
$$

Next, it is useful to employ the fourth order operator $D_{L}$ to cast the result of the variation into the form

$$
\partial_{t} S(g)=\int_{M}\left(-2 R D_{L} R+2 R R^{, a} R_{, a}+R^{2} \Delta R\right) d V(g)
$$

and observe that $\left(R^{2} R^{, a}\right)_{, a}=2 R R^{, a} R_{, a}+R^{2} \Delta R$. Thus, dropping the total derivative term, it follows immediately that

$$
\partial_{t} S(g)=-2 \int_{M} R D_{L} R d V(g)
$$

The operator $D_{L}$ is positive semi-definite and self-adjoint operator acting on functions on $M$. It can be further decomposed as $D_{L}=L^{\star} L$, where $L$ is a second order operator mapping complex valued functions to holomorphic tangent vector valued $(0,1)$-forms,

$$
L \varphi=\varphi^{, a c} g_{c \bar{b}} \frac{\partial}{\partial z^{a}} \otimes d \bar{z}^{b}
$$

and $L^{\star}$ denotes its adjoint. Then, using for simplicity the Hermitian product

$$
(\varphi, \psi)=\int_{M} \varphi(z, \bar{z}) \bar{\psi}(z, \bar{z}) d V(g),
$$


we have in total

$$
\partial_{t} S(g)=\partial_{t}(R, R)=-2\left(R, D_{L} R\right)=-2(L R, L R) \leq 0,
$$

showing that $S(g)$ is a Lyapunov functional that decreases monotonically along the Calabi flow.

The critical points correspond to extremal metrics on $M,[6,7]$, which according to the previous discussion satisfy the Euler-Lagrange equation $D_{L} R=0$, or equivalently the simpler equation $L R=0$ that describes its global solutions in a compact manifold $M$ without boundary. This, in turn implies that the extremal metrics, when they exist, are characterized by the condition that their curvature is such that $R^{a} \partial_{a}$ is a holomorphic vector field. In general, very little is known about the space of extremal Kähler metrics, but it is expected to have unique extremal metric in each Kähler class up to holomorphic transformations, apart from some special examples. Further analysis of the problem shows that if there exists a constant scalar curvature metric on $M$, then it achieves the absolute minimum value of $S(g)$. In this case, all extremal metrics have constant curvature and the absolute minimum value of the quadratic curvature functional that follows by application of Schwarz's inequality,

$$
S(g) \geq \frac{\left(\int_{M} R[g] d V(g)\right)^{2}}{\int_{M} d V(g)}
$$

is attained. However, there can be situations where extremal metrics exist on $M$ but they do not have constant scalar curvature; in fact, constant scalar curvature metrics and extremal metrics with non-constant scalar curvature do not co-exist in a single Kähler class.

Whenever the existence of a critical Kähler metric can be guaranteed, then the so called Futaki-Calabi obstructions determine the necessary and sufficient conditions for the existence of a constant scalar curvature metric in Kähler geometry, [8, 7]. Last, but not least, there are counter-examples of Kähler manifolds that do not admit any extremal metrics and, hence, $S(g)$ has no critical points; for, if extremal metrics exist they will necessarily be symmetric under a maximal compact subgroup of the holomorphic transformation group, but, in some cases, this fails to exist, [9]. Given these facts, it appears that the situation is rather complex in general and we will not attempt to fill in the technical details as they are beyond the scope of the present work; for an overview, see, for instance, $[10,11]$. Here, we will be mostly concerned with two-dimensional spaces with the topology of sphere, which admit a constant curvature metric - that of the round sphere - and permit the solutions of the Calabi flow to exist for sufficiently long time and converge to the canonical metric, $[12,13]$. The conditions for global existence and convergence of the Calabi flow to constant curvature metrics is a hard problem, in general, in higher dimensional spaces.

The physical relevance of the Calabi flow is not yet known for manifolds with arbitrary number of dimensions apart from the lowest dimensional case of one complex (or two real) dimensions. It is quite remarkable that the two-dimensional Calabi flow 
characterizes completely the solutions of four dimensional Einstein equations that describe gravitational radiation from bounded sources, using the general class of RobinsonTrautman metrics for space-times that admit a geodesic, shear and twist free diverging null congruence. In this context, $t$ is identified with the retarded time coordinate in space-time, as will be explained in detail in section 6 .

\subsection{A formal relation between the two flows}

There is a curious relation between the Ricci and Calabi flows on Kähler manifolds of arbitrary dimension that manifests by squaring the time evolution operator. In particular, taking the time derivative of the Ricci flow, we have

$$
\frac{\partial^{2}}{\partial t^{2}} g_{a \bar{b}}=-\frac{\partial}{\partial t} R_{a \bar{b}}=\partial_{a} \bar{\partial}_{b} \frac{\partial}{\partial t}(\log \operatorname{det} g)
$$

which in turn can be evaluated by noting $\partial_{t}(\log \operatorname{det} g)=g^{a \bar{b}} \partial_{t} g_{a \bar{b}}=-g^{a \bar{b}} R_{a \bar{b}}=-R$. Thus, in Kähler geometry, deformations of the metric flowing à la Ricci always imply the equivalent relation

$$
\frac{\partial^{2}}{\partial t^{2}} g_{a \bar{b}}=-\partial_{a} \bar{\partial}_{b} R
$$

Clearly, if the second derivative of the metric with respect to the Ricci time is identified with minus its first derivative with respect to the Calabi time,

$$
\frac{\partial^{2}}{\partial t_{\mathrm{R}}^{2}}=-\frac{\partial}{\partial t_{\mathrm{C}}},
$$

the two flows will be formally the same.

The procedure above looks similar in vain to the way of extracting the square root of the Schrödinger equation in supersymmetric quantum mechanics for a free particle (for a recent account see, for instance, [14], and references therein). In the latter case, this is practically achieved by considering a super-evolution equation

$$
\mathcal{D} \Psi=\mathcal{Q} \Psi
$$

where $\mathcal{D}$ is the supertime derivative with the defining property $\mathcal{D}^{2}=-i \partial / \partial t$ and $\mathcal{Q}$ is the supercharge of the theory satisfying the relation $\mathcal{D Q}+\mathcal{Q D}=0$. Then, the superevolution equation provides a consistent square root of the Schrödinger equation, because it implies

$$
-i \frac{\partial \Psi}{\partial t}=\mathcal{D}^{2} \Psi=\mathcal{D} \mathcal{Q} \Psi=-\mathcal{Q D} \Psi=-\mathcal{Q}^{2} \Psi=-H \Psi
$$

where $H=\mathcal{Q}^{2}$ is the Hamiltonian of the system. Of course, the order of the equations that govern geometric evolutions is different, and there are also non-linearities that make the problem at hand to be more complicated mathematically.

The analogy will be made more precise later by introducing anti-commuting parameters into the theory of geometric flows which promote time derivatives to super-evolution 
operators. This procedure, which works well in two dimensions, has far reaching consequences, as it will allow to cast the Calabi flow into zero curvature form and deduce its properties from those of the Ricci flow. It is for this reason that both flows are discussed in parallel while uncovering their algebraic structures in two dimensions. In the process, we will also be able to construct a hierarchy of higher order geometric evolution equations, together with a hierarchy of new infinite dimensional Lie algebras, which will be used for their zero curvature formulation. The general method works in the same way for higher dimensional Kähler manifolds, in that there is a squaring pattern for the time derivative operator that enables to connect consecutive flows, as above, but there is no zero curvature formulation for them above two dimensions. Thus, most of our work will be subsequently confined to two-dimensional spaces and postpone until the very end the derivation of some more general results that are valid in all dimensions.

\subsection{Landing in two dimensions}

Next, specializing to two dimensions and using a system of conformally flat (Kähler) coordinates,

$$
d s_{\mathrm{t}}^{2}=2 e^{\Phi(z, \bar{z} ; t)} d z d \bar{z}
$$

we find that the only non-vanishing component of the Ricci curvature tensor is $R_{z \bar{z}}=$ $-\partial \bar{\partial} \Phi$. Then, the two different geometric deformations take the following neat form for the conformal factor of the metric, $\Phi(z, \bar{z} ; t)$, respectively,

$$
\begin{array}{rlrl}
\text { Ricci flow : } & \partial_{t} \Phi & =\Delta \Phi, \\
\text { Calabi flow : } & \partial_{t} \Phi=-\Delta \Delta \Phi .
\end{array}
$$

The symbol $\Delta$ stands for the Laplace-Beltrami operator, with the analyst's sign,

$$
\Delta=e^{-\Phi} \partial \bar{\partial}
$$

which depends on $\Phi$ and introduces non-linearities into the system. These equations can be be defined on any Riemann surface of genus $g$, but for all practical purposes we can concentrate to spaces with spherical topology, $S^{2}$.

The dissipative character of these equations can be inferred from their linearization in the weak field approximation, where the curvature perturbations are assumed to be small. The reference metric around which such analysis should be carried, in order to make good physical and mathematical sense, depends on the flow. For the Ricci flow, it is appropriate to consider small fluctuations around the flat two-dimensional plane characterized by small and slowly varying values of $\Phi$. This region corresponds to $R_{\mu \nu} \simeq 0$, that is to the asymptotically free limit of a non-linear sigma model with target metric $g_{\mu \nu}$. In the language of renormalization group flows, it corresponds to the ultra-violet region when the curvature of the space is positive and to the infra-red region when the space is negatively curved. Then, since $\Phi \simeq 0$, the Ricci flow equation can 
be approximated by the linear heat equation $\partial_{t} \Phi=\partial \bar{\partial} \Phi$ in two-dimensional flat space, whose dissipative properties are well known.

On the other hand, for the Calabi flow, it is more appropriate to consider the round sphere $S^{2}$ as reference frame for introducing small curvature perturbations around it. The choice is essentially uniquely and dictated by the limiting extremal metric of the twodimensional Calabi flow and, therefore, one is led to examine fluctuations of the metric with $g=(1+f) g_{0}$, where $g_{0}$ corresponds to the line element $d s^{2}=2 R_{0}^{2} d z d \bar{z} /(1+z \bar{z})^{2}$ of a sphere of radius $R_{0}$ and $f$ is taken small. Let us consider, without great loss of generality, axially symmetric deformations of the round sphere, which are parametrized by Legendre polynomials $P_{l}(\xi)$ in the system of spherical coordinate $(\theta, \phi)$,

$$
d s_{\mathrm{t}}^{2}=R_{0}^{2}\left[1+\epsilon_{l}(t) P_{l}(\cos \theta)\right]\left(d \theta^{2}+\sin ^{2} \theta d \phi^{2}\right)
$$

with corresponding small parameters $\epsilon_{l}(t)$. Such configurations qualify as approximate solutions to the Calabi flow equation, as they preserve the volume to linear order in $\epsilon_{l}$,

$$
V_{\epsilon}=\int d V\left(g_{\epsilon}\right)=4 \pi R_{0}^{2}+2 \pi R_{0}^{2} \epsilon_{l}(t)(-1)^{l} \int_{-1}^{+1} d \xi P_{l}(\xi)=4 \pi R_{0}^{2}
$$

for all $l \geq 1$. In fact, the cases $l=0$ and $l=1$ will be omitted in the calculations, as they both represent round spheres. Thus, we assume that $l \geq 2$.

Then, to linear order in the perturbation parameters, the Calabi flow yields the following evolution

$$
\epsilon_{l}(t)=\epsilon_{l}(0) \exp \left(-\frac{t}{4 R_{0}^{4}} l\left(l^{2}-1\right)(l+2)\right) .
$$

The result, which approximates well the asymptotic behavior of the full non-linear evolution equation, when $t \rightarrow+\infty$, shows that all perturbations are damped exponentially fast and the configuration settles down to that of a round sphere. More general deformations corresponding to spherical harmonics $Y_{l}^{m}(\theta, \phi)$ with $m \neq 0$ can also be considered, but the results are essentially unaltered. All linearized solutions admit interesting physical interpretation in the context of multi-pole gravitational radiation from bounded sources, where they were first derived in the literature, as will be seen later in section 6 .

For comparison, a similar analysis is performed for the normalized Ricci flow, which is volume preserving and in two dimensions assumes the form

$$
\partial_{t} \Phi=\Delta \Phi+\frac{1}{R_{0}^{2}}
$$

when the space has spherical topology and its volume is taken equal to $4 \pi R_{0}^{2}$. In this case, one considers linearized perturbations about the round sphere of radius $R_{0}$, which constitutes the equilibrium state of the normalized flow. Using the same ansatz (2.34) as before, it turns out that the corresponding parameters evolve, to linear order, as

$$
\epsilon_{l}(t)=\epsilon_{l}(0) \exp \left(-\frac{t}{2 R_{0}^{2}}(l-1)(l+2)\right)
$$


and therefore, as $t \rightarrow+\infty$, the perturbations are also damped exponentially fast ${ }^{1}$. Comparison with the Calabi flow shows the same qualitative behavior, although in this case perturbations of a given angular momentum $l$ diminish at a slower pace; the dependence of $\epsilon_{l}(t)$ on $l$, being quadratic or quartic form, is a reflection of the corresponding evolution equations, being second and fourth order, respectively.

It is natural to expect that the dissipative properties of the linearized flows will be also shared by the full non-linear parabolic equations of second and fourth order, respectively, as they do. The difficulty with the non-linearities is that they prevent the construction of explicit solutions, apart from a few isolated simple examples. In most cases, it is quite difficult to introduce ansatz that effectively reduce the non-linear equations to a simpler system of evolution equations for a finite number of moduli. The Ricci flow appears to be more tractable by such methods, since there is already a good number of simple (yet interesting) exact solutions. The situation is more primitive for the Calabi flow as only a handful of exact solutions is known.

\section{Algebraic framework}

Our primary aim in the following is to device a suitable algebraic method for establishing the integrability of the two different geometric deformations in two dimensions. It proves useful to sketch first the algebraic techniques that have already been used successfully for the formal integration of the Ricci flow and then extend their domain of applicability to the Calabi flow and further beyond.

The notion of continual Lie algebras provides the main framework for the systematic formulation of the geometric flows as Toda field equations in two dimensions. The key idea is to treat the deformation variable $t$ as a continuous "Dynkin index" of an appropriately chosen infinite dimensional algebra and rewrite the Ricci and Calabi flows as zero curvature conditions in the two-dimensional system of conformally flat coordinates. Here, we present the basic definitions of continual Lie algebras and the Toda systems associated to them. We also review the general algorithm for obtaining their general solutions in terms of two dimensional free fields, using group theoretical techniques for the Bäcklund transformations. The specific choice of continual algebras that enable to cast different geometric flows into zero curvature form will be determined in the following sections together with the formal power series solutions of the equations.

\footnotetext{
${ }^{1}$ Of course, the time of the normalized flow depends logarithmically on the time of the unnormalized flow, as $-R_{0}^{2} \log (-t)$, according to the general relation between the two; thus, a spherical configuration that starts from very large size in the ultra-violet region $t \rightarrow-\infty$ and diminishes linearly to zero size at $t=0$ by the Ricci flow, will correspond to a volume preserving deformation of the sphere under the normalized Ricci flow that now runs from $-\infty$ to $+\infty$ and tends to become round exponentially fast.
} 


\subsection{Continual Lie algebras}

The basic theory of continual Lie algebras is formulated by introducing a system of Cartan-Weyl generators $\left\{H(t), X^{ \pm}(t)\right\}$ that depend on a continuous variable $t$ and satisfy the system of commutation relations, $[15,16]$,

$$
\begin{aligned}
& {\left[X^{+}(t), X^{-}\left(t^{\prime}\right)\right]=S\left(t, t^{\prime}\right) H\left(t^{\prime}\right), \quad\left[H(t), H\left(t^{\prime}\right)\right]=0} \\
& {\left[H(t), X^{ \pm}\left(t^{\prime}\right)\right]= \pm K\left(t, t^{\prime}\right) X^{ \pm}\left(t^{\prime}\right)}
\end{aligned}
$$

$K\left(t, t^{\prime}\right)$ is called the Cartan kernel of the algebra and it generalizes the Cartan matrix $K_{i j}$ of simple Lie algebras to the continuous case, whereas the typical choice for $S$ is $S\left(t, t^{\prime}\right)=$ $\delta\left(t-t^{\prime}\right)$, which corresponds to $\delta_{i j}$ in the case of discrete roots. This algebraic structure can be alternatively defined by smearing the Cartan-Weyl generators with arbitrary functions $\varphi(t)$ of compact support, as in the theory of distributions. Thus, setting

$$
A(\varphi)=\int d t \varphi(t) A(t)
$$

the basic commutation relations assume the form

$$
\begin{aligned}
& {\left[X^{+}(\varphi), X^{-}(\psi)\right]=H(S(\varphi, \psi)), \quad[H(\varphi), H(\psi)]=0,} \\
& {\left[H(\varphi), X^{ \pm}(\psi)\right]= \pm X^{ \pm}(K(\varphi, \psi))}
\end{aligned}
$$

In general, the operators $K$ and $S$ are thought to be bilinear maps on the vector space of functions $\varphi(t)$ satisfying the relations

$$
K(\varphi, K(\psi, \chi))=K(\psi, K(\varphi, \chi)), \quad S(\varphi, K(\psi, \chi))=S(K(\psi, \varphi), \chi)
$$

for consistency with the Jacobi identities. Here, we only need to consider the case $K(\varphi, \psi)=(K \varphi) \psi$ and $S(\varphi, \psi)=S(\varphi \psi)$ prescribed by linear operators $K$ and $S$, which always ensure the validity of the Jacobi identities. The corresponding continual Lie algebra will be denoted by $\mathcal{G}(K, S)$, but it can be easily shown that $\mathcal{G}(K, S) \simeq \mathcal{G}(\tilde{K}, 1)$ with $\tilde{K}=K S$; for it suffices to define a new basis of Cartan generators $\tilde{H}(\varphi)=H(S \varphi)$. Therefore, for all practical purposes, the continual Lie algebras can be brought into standard form, which is abbreviated to $\mathcal{G}(K)$, setting $S=1$ and dropping the tilde from $K$. From now on we will use this notation unless stated otherwise. The Cartan kernel $K$ can be symmetrizable, but for the purposes of the present work we will also allow for algebras with anti-symmetric Cartan kernels $K\left(t, t^{\prime}\right)=-K\left(t^{\prime}, t\right)$. In fact, the geometric flows turn out to be naturally related to continual Lie algebras with anti-symmetric kernels, as will be shown later in detail.

The continual Lie algebras $\mathcal{G}(K)$ are infinite dimensional $Z$-graded algebras with direct decomposition into subspaces

$$
\mathcal{G}(K)=\oplus_{n \in Z} \mathcal{G}_{n}
$$


The Cartan-Weyl generators $H$ and $X^{ \pm}$span the components $\mathcal{G}_{0}$ and $\mathcal{G}_{ \pm 1}$, respectively, thus forming the local part of the algebra $\mathcal{G}_{-1} \oplus \mathcal{G}_{0} \oplus \mathcal{G}_{+1}$, whereas all other elements of it can be constructed recursively by taking successive commutators of the basic generators so that $\mathcal{G}_{n}=\left[\mathcal{G}_{n-1}, \mathcal{G}_{+1}\right]$ for all $n>1$ and $\mathcal{G}_{n}=\left[\mathcal{G}_{n+1}, \mathcal{G}_{-1}\right]$ for all $n<-1$. The growth of the algebra which is characterized by the dimension of the subspaces $\mathcal{G}_{n}$ relative to the dimension of $\mathcal{G}_{0}$ can vary accordingly depending on the choice of $K$. The derivation of all commutation relations of the algebra $\mathcal{G}(K)$ is not an easy task as there is no known analogue of the Serre relations in the general case. As a result, the complete description of $\mathcal{G}(K)$ is still lacking for arbitrary $K$, and in particular for those cases that will be encountered in the algebraic description of geometric flows. Nevertheless, the zero curvature formulation of the flows, and their integration, will be solely based on the local part of the algebras without ever requiring knowledge of the full structure of $\mathcal{G}(K)$ in either case.

\subsection{Associated Toda systems}

The Toda system associated to any continual Lie algebra with Cartan operator $K$ is defined to be

$$
\partial \bar{\partial} \Phi(z, \bar{z} ; t)=\int d t^{\prime} K\left(t^{\prime}, t\right) e^{\Phi\left(z, \bar{z} ; t^{\prime}\right)} .
$$

$\Phi(z, \bar{z} ; t)$ can be viewed as a one-parameter family of two dimensional fields that depend on a continuous variable $t$ rather than on discrete indices $i,[15,16]$. As such, they generalize the Toda field equations associated to simple Lie algebras with Cartan matrix $K_{i j}$ to the case of infinite dimensional algebras with a continuous system of roots, at least formally. In analogy to ordinary Toda systems, this equation is cast into zero curvature form

$$
\left[\partial+A_{+}(z, \bar{z}), \bar{\partial}+A_{-}(z, \bar{z})\right]=0
$$

using appropriate gauge connections $A_{ \pm}$taking their values in the local part $\mathcal{G}_{0} \oplus \mathcal{G}_{ \pm 1}$ of the algebra $\mathcal{G}(K)$.

More precisely, let us consider the general ansatz for the two-dimensional gauge connections

$$
A_{ \pm}(z, \bar{z})=H\left(u_{ \pm}\right)+X^{ \pm}\left(f_{ \pm}\right)
$$

which depend on functions $u_{ \pm}$and $f_{ \pm}$of $z, \bar{z}$ and $t$. Some of these functions are superfluous and they can be eliminated by employing the gauge invariance of the zero curvature condition under the transformations

$$
A_{+} \rightarrow g^{-1}\left(\partial+A_{+}\right) g, \quad A_{-} \rightarrow g^{-1}\left(\bar{\partial}+A_{-}\right) g
$$

with arbitrary group elements $g=\exp H(\varphi)$. As it turns out, it is always possible to choose the gauge

$$
A_{+}=H(\Psi)+X^{+}(1), \quad A_{-}=X^{-}\left(e^{\Phi}\right)
$$


and then use the commutation relations of the algebra in order to obtain the following system of equations from the zero curvature condition,

$$
\bar{\partial} \Psi=e^{\Phi}, \quad \partial \Phi=K(\Psi)
$$

Elimination of the function $\Psi$ leads to the continual Toda field equation

$$
\partial \bar{\partial} \Phi=K\left(e^{\Phi}\right)
$$

which is the smeared form of equation (3.6) above.

The general solution is obtained in analogy to ordinary Toda systems (for a review, see, for instance, [17]), by introducing a normalized highest weight state $\mid t>$ that depends on the continuous variable $t$,

$$
X^{+}\left(t^{\prime}\right)|t>=0, \quad<t| X^{-}\left(t^{\prime}\right)=0, \quad H\left(t^{\prime}\right)\left|t>=\delta\left(t-t^{\prime}\right)\right| t>
$$

with $\langle t \mid t\rangle=1$. Then, the one-parameter family of two dimensional free fields with the decomposition

$$
\Phi_{0}(z, \bar{z} ; t)=f(z ; t)+\bar{f}(\bar{z} ; t)
$$

following from $\partial \bar{\partial} \Phi_{0}=0$, is used to express the Toda field configurations in the form, $[15,16]$,

$$
\Phi(z, \bar{z} ; t)=\Phi_{0}(z, \bar{z} ; t)-K\left(\log <t\left|M_{+}^{-1}(z) M_{-}(\bar{z})\right| t>\right)
$$

where $M_{ \pm}$are given by the path-ordered exponentials of the Lie algebra elements in $\mathcal{G}_{ \pm 1}$, respectively,

$$
\begin{aligned}
& M_{+}(z)=\mathcal{P} \exp \left(\int^{z} d z^{\prime} \int d t^{\prime} e^{f\left(z^{\prime} ; t^{\prime}\right)} X^{+}\left(t^{\prime}\right)\right) \\
& M_{-}(\bar{z})=\mathcal{P} \exp \left(\int^{\bar{z}} d \bar{z}^{\prime} \int d t^{\prime} e^{\bar{f}\left(\bar{z}^{\prime} ; t^{\prime}\right)} X^{-}\left(t^{\prime}\right)\right) .
\end{aligned}
$$

This formula summarizes the Bäcklund transformations of continual Toda systems, thus providing a free field realization of their general solution.

The details can be worked out by expanding $M_{ \pm}$in power series and compute the expectation value of their product in the highest weight state, as

$$
\begin{aligned}
&<t \mid M_{+}^{-1} M_{-} \mid t>=1+\sum_{n=1}^{\infty}(-1)^{n} \int^{z} d z_{1} \cdots \int^{z_{n-1}} d z_{n} \int^{\bar{z}} d \bar{z}_{1} \cdots \int^{\bar{z}_{n-1}} d \bar{z}_{n} \times \\
& \times \int \prod_{i=1}^{n} d t_{i} \int \prod_{i=1}^{n} d t_{i}^{\prime} \exp f\left(z_{i} ; t_{i}\right) \exp \bar{f}\left(\bar{z}_{i} ; t_{i}^{\prime}\right) D_{t}^{\left\{t_{1}, \cdots, t_{n} ; t_{1}^{\prime}, \cdots, t_{n}^{\prime}\right\}}
\end{aligned}
$$

with the ordering $z \geq z_{1} \geq \cdots \geq z_{n-1} \geq z_{n}$ and likewise for the $\bar{z}$ 's. The structure of the algebra $\mathcal{G}(K)$ is fully encoded into the elements

$$
D_{t}^{\left\{t_{1}, t_{2}, \cdots, t_{n} ; t_{1}^{\prime}, t_{2}^{\prime}, \cdots, t_{n}^{\prime}\right\}}=<t\left|X^{+}\left(t_{1}\right) X^{+}\left(t_{2}\right) \cdots X^{+}\left(t_{n}\right) X^{-}\left(t_{n}^{\prime}\right) \cdots X^{-}\left(t_{2}^{\prime}\right) X^{-}\left(t_{1}^{\prime}\right)\right| t>
$$


that depend on the choice of Cartan operator $K$. They are determined using the recursive relations

$$
D_{t}^{\left\{t_{1}, t_{2}, \cdots, t_{n} ; t_{1}^{\prime}, t_{2}^{\prime}, \cdots, t_{n}^{\prime}\right\}}=\sum_{j=1}^{n} \delta\left(t_{n}-t_{j}^{\prime}\right)\left(\delta\left(t-t_{j}^{\prime}\right)-\sum_{k=1}^{j-1} K\left(t_{j}^{\prime}, t_{k}^{\prime}\right)\right) D_{t}^{\left\{t_{1}, \cdots, t_{n-1} ; t_{1}^{\prime}, \cdots, \hat{t}_{j}^{\prime}, \cdots, t_{n}^{\prime}\right\}}
$$

with $D_{t}^{\left\{t_{1} ; t_{1}^{\prime}\right\}}=\delta\left(t-t_{1}\right) \delta\left(t-t_{1}^{\prime}\right)$. Here, $\hat{t}_{j}^{\prime}$ is used to denote that $t_{j}^{\prime}$ has been omitted by contraction with $t_{m}$ while shifting $X^{+}$'s to the right and $X^{-}$'s to the left.

Thus, straightforward extension of the group theoretical methods used for the integration of Toda systems for simple Lie algebras allows to construct the general solution of continual Toda field equations and obtain a formal infinite power series expansion around free field configurations. In regions where the exponential self-interaction becomes negligible one simply has $\Phi \simeq \Phi_{0}$ for the corresponding Toda fields. Such formal expansion may or may not converge in the strict mathematical sense, but there is no general way to decide on such issues at the moment.

The simplest and mostly studied example of this kind is provided by the so called heavenly equation, which is the following non-linear equation for a field $\Phi(z, \bar{z} ; t)$,

$$
\frac{\partial^{2}}{\partial t^{2}} e^{\Phi}=-\partial \bar{\partial} \Phi
$$

This equation is not parabolic, unlike the equations associated to geometric evolutions, for it involves second derivatives in space and time. It arises in the context of fourdimensional hyper-Kähler manifolds that admit a non-triholomorphic isometry associated to a rotational $S^{1}$ action, and describes the self-duality condition on the Riemann curvature tensor of the metric in adapted coordinates, [18]. In the present context, it is identified with the Toda field equation for the large $N$ limit of the $S U(N)$ algebra, where the Cartan matrix $K_{i j}=2 \delta_{i, j}-\delta_{i+1, j}-\delta_{i, j+1}$ is replaced by the kernel $K\left(t, t^{\prime}\right)=-\delta^{\prime \prime}\left(t-t^{\prime}\right)$ using a continuous parameter $t$ to label the vertices of the Dynkin diagram, [19]. Thus, the corresponding Cartan operator is $K=-\partial^{2} / \partial t^{2}$. Integration of the heavenly equation by group theoretical methods has been carried out, as for Toda theories, and various geometrically interesting solutions have been studied explicitly; the examples include the Eguchi-Hanson, Taub-NUT and Atiyah-Hitchin metrics, which all admit at least one non-triholomorphic $S^{1}$ action. In that context, the validity of the formal power series expansion of the general solution in terms of free fields has been tested in several cases, and the interested reader is referred to the literature, [20], for details.

\subsection{Generalization to supercontinual Lie algebras}

Next, we introduce the more general class of supercontinual Lie algebras which are defined by the basic system of commutation relations

$$
\begin{aligned}
& {\left[X^{+}(\mathcal{F}), X^{-}(\mathcal{G})\right]=H(\mathcal{F} \mathcal{G}), \quad[H(\mathcal{F}), H(\mathcal{G})]=0} \\
& {\left[H(\mathcal{F}), X^{ \pm}(\mathcal{G})\right]= \pm X^{ \pm}((K \mathcal{F}) \mathcal{G})}
\end{aligned}
$$


for the generators $H$ and $X^{ \pm}$. The difference with the ordinary case is that the smearing functions $\mathcal{F}$ are taken to be super-functions on $R^{1 \mid 1}$ superspace with coordinate $T=(t, \theta)$ having $\theta^{2}=0$. Likewise, the generators $H(T)$ and $X^{ \pm}(T)$ are not bosonic but they also admit an expansion in $R^{1 \mid 1}$ superspace. However, the smeared form of the generators $H(\mathcal{F})$ and $X^{ \pm}(\mathcal{F})$ is considered to be bosonic so that they possess commutation relations rather than anti-commutation relations. Thus, in effect, supercontinual Lie algebra provide a special class of infinite dimensional Lie algebras that can be brought into a standard Cartan form with the aid of a Grassmann variable $\theta$.

The corresponding Cartan operator $K$ can be an ordinary linear operator which is defined on the space of functions of a continuous variable $t$ and acts separately on each component of the smearing super-functions $\mathcal{F}$. More generally, it can be a super-operator acting linearly on the space of super-functions $\mathcal{F}(T)$. In either case, the Cartan operator has to satisfy the relations

$$
(K \mathcal{F}) \mathcal{G}=\mathcal{G}(K \mathcal{F}), \quad(K \mathcal{F})(K \mathcal{G})=(K \mathcal{G})(K \mathcal{F})
$$

for all $\mathcal{F}$ and $\mathcal{G}$, as follow from the Jacobi identity for the two triplets $\left(H, X^{+}, X^{-}\right)$ and $\left(H, H, X^{ \pm}\right)$. These put no special restrictions on the choices that are available, and, therefore, the class of super-continual Lie algebras is well defined for all $K$. A particularly interesting example, which is relevant for the algebraic description of the Calabi flow, corresponds to the odd choice $K=\partial / \partial \theta-\theta \partial / \partial t$, as will be seen later. Other choices are also possible, but their physical relevance is not known in general.

The smearing super-functions $\mathcal{F}$ can be expanded in components as

$$
\mathcal{F}(T)=\varphi_{0}(t)+\theta \varphi_{1}(t)
$$

using the superspace coordinates $T=(t, \theta)$. Likewise, the generators of the algebra can be viewed as being parametrized by a continuous super-time parameter $T$ with respect to which they also admit expansion into components,

$$
A(T)=A_{0}(t)+\theta A_{1}(t)
$$

with $A$ being either $H$ or $X^{ \pm}$. By definition, the two components $\varphi_{0}(t)$ and $\varphi_{1}(t)$ are even functions taking values in ordinary numbers, and similarly $A_{0}(t)$ and $A_{1}(t)$ are also even. The smearing is defined by complete integration in $R^{1 \mid 1}$ superspace with measure $d T=d t d \theta$, as follows,

$$
A(\mathcal{F})=\int A(T) \mathcal{F}(T) d T=A_{0}\left(\varphi_{1}\right)+A_{1}\left(\varphi_{0}\right),
$$

using the known identities $\int d \theta=0$ and $\int \theta d \theta=1$. Thus, all such $A(\mathcal{F})$ are even, as required. Then, the structure of the commutation relations can be worked out in components for the bosonic generators $H_{i}(\varphi)$ and $X_{i}^{ \pm}(\varphi)$ with $i=0,1$, depending on the form of $K$.

Toda systems associated to such algebraic structures can be defined as usual by the zero curvature form $\left[\partial+A_{+}(z, \bar{z}), \bar{\partial}+A_{-}(z, \bar{z})\right]=0$, choosing

$$
A_{+}=H(\mathcal{G})+X^{+}(1), \quad A_{-}=X^{-}\left(e^{\mathcal{F}}\right)
$$


for the gauge connections. Then, if the super-functions $\mathcal{F}$ and $\mathcal{G}$ are related to each other as $\partial \mathcal{F}=K(\mathcal{G})$, the corresponding Toda field equation will be

$$
\partial \bar{\partial} \mathcal{F}=K\left(e^{\mathcal{F}}\right)
$$

Expanding $\mathcal{F}$ in components and comparing the even and odd parts of the equation yields an equivalent description of the associated Toda system. The general solution can also be obtained in this case by straightforward generalization of the group theoretical methods developed for ordinary Toda systems. Further details will be given later for the particular example of the Calabi flow.

Other possibilities may arise, in principle, by considering $\mathcal{F}$ as super-functions on $R^{1 \mid N}$ superspace, according to our general discussion. The structure of the associated supercontinual Lie algebras can also be worked out in component form, but become more complicated for higher vales of $N$.

\section{Ricci flow}

According to the general discussion in section 2, the Ricci flow equation in two dimensions assumes the following simple form in a system of conformally flat coordinates $(z, \bar{z})$,

$$
\partial \bar{\partial} \Phi(z, \bar{z} ; t)=\partial_{t} e^{\Phi(z, \bar{z} ; t)}
$$

where $\Phi$ is the conformal factor of the metric undergoing continuous deformations. As such, it admits a natural algebraic interpretation in the framework of Toda field equations for the choice of continual Lie algebra with Cartan operator $K=\partial / \partial t$. In particular, following [21, 22], we consider the continual Lie algebra whose local part satisfies the commutation relations, in smeared form,

$$
\begin{aligned}
& {\left[X^{+}(\varphi), X^{-}(\psi)\right]=H(\varphi \psi), \quad[H(\varphi), H(\psi)]=0} \\
& {\left[H(\varphi), X^{ \pm}(\psi)\right]= \pm X^{ \pm}\left(\varphi^{\prime} \psi\right) .}
\end{aligned}
$$

Then, the two-dimensional Ricci flow, which is viewed as Toda system for this algebra, $[21,22]$, admits the zero curvature formulation

$$
\left[\partial+A_{+}(z, \bar{z}), \bar{\partial}+A_{-}(z, \bar{z})\right]=0
$$

where $A_{ \pm}$take values in the local part as

$$
A_{+}=H(\Psi)+X^{+}(1), \quad A_{-}=X^{-}\left(e^{\Phi}\right) .
$$

Also, following the general discussion in section 3, we find that $\partial \Phi=\partial_{t} \Psi$ and $\bar{\partial} \Psi=\exp \Phi$ and, hence, $\Phi$ satisfies the Ricci flow equation (4.1) after eliminating $\Psi$.

The main ingredient in this particular formulation of the Ricci flow is the use of a continual Lie algebra to incorporate the deformation variable $t$ into its root system. Thus, 
the equation is integrable in two dimensions using flat space coordinates $(z, \bar{z})$, without reaching a contradiction of terms with the dissipative behavior in time. In this context, the parabolic nature of the flow, which is first order in $t$, is held responsible for the novel structure of the underlying Lie algebra with anti-symmetric Cartan kernel

$$
K\left(t, t^{\prime}\right)=-\frac{\partial}{\partial t} \delta\left(t-t^{\prime}\right)
$$

Such algebras have been studied very little in the mathematics literature, but it has already been noted that $\mathcal{G}(\partial / \partial t)$ exhibits exponentially fast growth beyond its local part. As a result, its complete structure is still lacking and there is no framework in which to realize transformation rules for all the generators. Also, the precise meaning of integrability for systems associated to such algebras should be understood better in the future, and in connection with the explicit construction of their conservation laws in twodimensional space. The fact is that they become relevant for the algebraic description of certain dynamical problems with the Ricci flow being the first example of this kind. The Calabi flow also falls in this category for appropriate choice of Cartan operator in the class of supercontinual Lie algebras, as will be seen later in detail.

Next, we apply the general formalism to describe the solutions of the two-dimensional Ricci flow equation in terms of a one-parameter family of free fields $\Phi_{0}(z, \bar{z} ; t)=f(z ; t)+$ $\bar{f}(\bar{z} ; t)$. The general expression $(3.15)$ specializes to

$$
\Phi(z, \bar{z} ; t)=\Phi_{0}(z, \bar{z} ; t)-\partial_{t}\left(\log <t\left|M_{+}^{-1}(z) M_{-}(\bar{z})\right| t>\right)
$$

where $M_{ \pm}$are the path ordered exponentials (3.16) that should be evaluated for the particular algebra $\mathcal{G}(\partial / \partial t)$. This provides the solution in closed form by group theoretical methods, but it is very implicit. In practice, one should evaluate recursively the elements $D_{t}^{\left\{t_{1}, t_{2}, \cdots, t_{n} ; t_{1}^{\prime}, t_{2}^{\prime}, \cdots, t_{n}^{\prime}\right\}}$ and substitute them into the general power series expansion (3.17).

Explicit calculation yields in this case the following results, up to $n=3$, [21],

$$
\begin{aligned}
& D_{t}^{\left\{t_{1} ; t_{1}^{\prime}\right\}}=\delta\left(t-t_{1}\right) \delta\left(t_{1}-t_{1}^{\prime}\right) \\
& D_{t}^{\left\{t_{1}, t_{2} ; t_{1}^{\prime}, t_{2}^{\prime}\right\}}=\delta\left(t-t_{1}\right) \delta\left(t_{1}-t_{1}^{\prime}\right) \delta\left(t_{2}-t_{2}^{\prime}\right)\left(2 \delta\left(t-t_{2}\right)-\partial_{t} \delta\left(t-t_{2}\right)\right) \\
& D_{t}^{\left\{t_{1}, t_{2}, t_{3} ; t_{1}^{\prime}, t_{2}^{\prime}, t_{3}^{\prime}\right\}}=\delta\left(t-t_{1}\right) \delta\left(t_{1}-t_{1}^{\prime}\right) \delta\left(t-t_{3}\right) \delta\left(t_{2}-t_{3}^{\prime}\right) \delta\left(t_{3}-t_{2}^{\prime}\right) \times \\
& \quad \times\left(2 \delta\left(t-t_{2}\right)-\partial_{t} \delta\left(t-t_{2}\right)\right)+ \\
& +\delta\left(t-t_{1}\right) \delta\left(t_{1}-t_{1}^{\prime}\right) \delta\left(t_{2}-t_{3}^{\prime}\right) \delta\left(t_{3}-t_{2}^{\prime}\right) \times \\
& \quad \times\left(\delta\left(t-t_{3}\right)-\partial_{t} \delta\left(t-t_{3}\right)\right)\left(2 \delta\left(t-t_{2}\right)-\partial_{t} \delta\left(t-t_{2}\right)\right)+ \\
& + \\
& \quad \delta\left(t-t_{1}\right) \delta\left(t_{1}-t_{1}^{\prime}\right) \delta\left(t_{2}-t_{2}^{\prime}\right) \delta\left(t_{3}-t_{3}^{\prime}\right) \times \\
& \quad \times\left(2 \delta\left(t-t_{2}\right)-\partial_{t} \delta\left(t-t_{2}\right)\right)\left(\delta\left(t-t_{3}\right)-\partial_{t} \delta\left(t-t_{3}\right)-\partial_{t_{2}} \delta\left(t_{2}-t_{3}\right)\right)
\end{aligned}
$$

and so on for higher $n$. The expressions become considerably longer beyond $n=3$ and they are not included here. 
In special cases, where the corresponding geometric deformations are axially symmetric, the solutions can be taken to depend only on the combination of coordinates $z+\bar{z}:=Y$. Then, for free field configurations of the form $\Phi_{0}(Y ; t)=c Y+d(t)$, the systematic expansion of the path ordered exponential leads to the power series solution

$$
\begin{gathered}
\Phi=\Phi_{0}+\frac{1}{(1 ! c)^{2}} \partial_{t} e^{\Phi_{0}}+\frac{1}{\left(2 ! c^{2}\right)^{2}} \partial_{t}\left(e^{\Phi_{0}} \partial_{t} e^{\Phi_{0}}\right)+\frac{1}{\left(3 ! c^{3}\right)^{2}} \partial_{t}\left(3 e^{\Phi_{0}}\left(\partial_{t} e^{\Phi_{0}}\right)^{2}+e^{2 \Phi_{0}} \partial_{t}^{2} e^{\Phi_{0}}\right) \\
+\frac{1}{\left(4 ! c^{4}\right)^{2}} \partial_{t}\left(e^{3 \Phi_{0}} \partial_{t}^{3} e^{\Phi_{0}}+17 e^{2 \Phi_{0}}\left(\partial_{t} e^{\Phi_{0}}\right)\left(\partial_{t}^{2} e^{\Phi_{0}}\right)+18 e^{\Phi_{0}}\left(\partial_{t} e^{\Phi_{0}}\right)^{3}\right) \\
+\frac{1}{\left(5 ! c^{5}\right)^{2}} \partial_{t}\left(e^{4 \Phi_{0}} \partial_{t}^{4} e^{\Phi_{0}}+36 e^{3 \Phi_{0}}\left(\partial_{t} e^{\Phi_{0}}\right)\left(\partial_{t}^{3} e^{\Phi_{0}}\right)+35 e^{3 \Phi_{0}}\left(\partial_{t}^{2} e^{\Phi_{0}}\right)^{2}\right. \\
\left.+324 e^{2 \Phi_{0}}\left(\partial_{t} e^{\Phi_{0}}\right)^{2}\left(\partial_{t}^{2} e^{\Phi_{0}}\right)+180 e^{\Phi_{0}}\left(\partial_{t} e^{\Phi_{0}}\right)^{4}\right)+\cdots
\end{gathered}
$$

by evaluating the contribution of all terms in the expansion up to order $n=5$ included. It should be understood as a power series expansion of $\exp \Phi$ around the free field configuration $\exp \Phi_{0}$ that becomes very small when $Y \rightarrow \pm \infty$ for $c<0$ or $>0$, respectively. It can also be verified directly that this expansion satisfies the Ricci flow equation order by order in powers of $\exp \Phi_{0}$.

The validity of the formal power series expansion and its convergence have be tested in various examples of axially symmetric geometric deformation that are known in closed form using more direct (mini-superspace) methods. A notable example is the sausage model that corresponds to the choice of conformal factor, [23],

$$
e^{\Phi(Y ; t)}=\frac{2}{a(t)+b(t) \cosh 2 Y} \quad \text { with } \quad 0 \leq X \leq 2 \pi, \quad-\infty<Y<+\infty
$$

while $t$ is running from $-\infty$ to 0 and

$$
a(t)=-\gamma \operatorname{coth}(2 \gamma t), \quad b(t)=-\frac{\gamma}{\sinh (2 \gamma t)} .
$$

Note that $a \geq b \geq 0$ since $t$ assumes only negative values; $t \rightarrow-\infty$ corresponds to the asymptotically free ultra-violet limit of the $O(3)$ sigma model in two dimensions. This solution describes a family of axially symmetric deformations of the round 2 -sphere for all values of the parameter $\gamma \geq 0$. It fits the expansion (4.8) in power of $\exp (-2 Y)$ for large $Y,[21]$, provided that the free field is chosen to be $\Phi_{0}(Y ; t)=c Y+d(t)$ with

$$
c=-2, \quad d(t)=\log \left(-\frac{4}{\gamma} \sinh (2 \gamma t)\right) .
$$

Thus, there is mounting confidence that the formal manipulations used for the integration of the Ricci flow indeed make good sense. Further details can be found in the published works, $[21,22]$. The only drawback of the present formalism is the inability to prescribe directly the geometric shapes of space from different choices of free fields. 


\section{Calabi flow}

Next, we turn to the two-dimensional Calabi flow, which will be shown to admit zero curvature formulation for appropriately chosen gauge connections. The method of investigation involves the introduction of anti-commuting variables following a simple observation that formally relates it to the Ricci flow. Then, we give full justification for the choice of the infinite dimensional algebra that enables to cast the Calabi flow in integrable form, as Toda system, and extend the validity of the group theoretical methods introduced earlier in order to describe (at least formally) the general solution in terms of free fields, as for the Ricci flow. The results described below complement earlier work on the subject by providing full justification of the seemingly ad hoc choices made in reference [24] and go far beyond it in some directions.

\subsection{Calabi flow as super-evolution}

According to the general discussion found in section 2, the two-dimensional Calabi flow assumes explicitly the form

$$
\partial_{t} e^{\Phi}=-\partial \bar{\partial}\left(e^{-\Phi} \partial \bar{\partial} \Phi\right)
$$

in a system of conformally flat coordinates with conformal factor $\Phi(z, \bar{z} ; t)$ varying with time $t$. Specializing an earlier observation, it is instructive to compared it to the twodimensional Ricci flow equation (4.1), which is equivalently stated as

$$
\frac{\partial^{2}}{\partial t^{2}} e^{\Phi}=\partial \bar{\partial}\left(e^{-\Phi} \partial \bar{\partial} \Phi\right)
$$

after taking its derivative with respect to $t$. Indeed, the Calabi flow appears to be formally related to the Ricci flow by taking the square root of its evolution operator $\partial / \partial t$, up to a sign.

This simple correspondence motivates us to introduce an anti-commuting variable $\theta$, with $\theta^{2}=0$, as the supersymmetric partner of the deformation variable $t$ and write down the following abstract equation

$$
\partial \bar{\partial} \mathcal{F}=\mathcal{D}_{T} \exp \mathcal{F}
$$

where

$$
\mathcal{F}(z, \bar{z} ; t, \theta)=\Phi(z, \bar{z} ; t)+\theta \Psi(z, \bar{z} ; t)
$$

is a mixed superfield with bosonic components $\Phi, \Psi$ and

$$
\mathcal{D}_{T}=\frac{\partial}{\partial \theta}-\theta \frac{\partial}{\partial t}
$$

is the associated super-derivative in $R^{1 \mid 1}$ superspace with coordinates $T=(t, \theta)$. It satisfies the relation $\mathcal{D}_{T}^{2}=-\partial / \partial t$, whereas the complementary super-derivative operator $\partial / \partial \theta+\theta \partial / \partial t$ squares to $\partial / \partial t$, since it relates $t$ to $-t$. The equation above can be 
viewed as some short of super-evolution equation of Ricci type with respect to the supertime $T$. Its precise content can be extracted by working in components. Expanding $\exp \mathcal{F}=(1+\theta \Psi) \exp \Phi$ and comparing the even and odd parts, it readily follows that

$$
e^{\Phi} \Psi=\partial \bar{\partial} \Phi, \quad \partial_{t} e^{\Phi}=-\partial \bar{\partial} \Psi
$$

Subsequently, by eliminating $\Psi$, it follows that $\Phi$ satisfies the Calabi flow equation (5.1) above. Thus, by taking the square root of the time derivative operator allows to connect the two distinct classes of geometric deformations of second and fourth, respectively, via super-evolution, as was anticipated before on general grounds.

This simple result has far reaching consequences, as it allows to prove integrability of the two-dimensional Calabi flow by viewing it as Toda field equation for a particular supercontinual algebra with odd Cartan operator $K=\mathcal{D}_{T}$, in analogy to the Ricci flow that corresponds to the choice $K=\partial / \partial t$. More precisely, we are led to introduce an algebra whose local part satisfies the commutation relations

$$
\begin{aligned}
& {\left[X^{+}(\mathcal{F}), X^{-}(\mathcal{G})\right]=H(\mathcal{F} \mathcal{G}), \quad[H(\mathcal{F}), H(\mathcal{G})]=0} \\
& {\left[H(\mathcal{F}), X^{ \pm}(\mathcal{G})\right]= \pm X^{ \pm}\left(\left(\mathcal{D}_{T} \mathcal{F}\right) \mathcal{G}\right)}
\end{aligned}
$$

It defines a supercontinual Lie algebra in the nomenclature introduced earlier.

Then, following the general discussion in section 3 , we write down the zero curvature condition

$$
\left[\partial+A_{+}(z, \bar{z}), \bar{\partial}+A_{-}(z, \bar{z})\right]=0,
$$

where the connections $A_{ \pm}$take values in the local part of the algebra, as

$$
A_{+}=H(\mathcal{G})+X^{+}(1), \quad A_{-}=X^{-}(\exp \mathcal{F})
$$

The superfield $\mathcal{F}$ satisfies the flow equation (5.3), provided that $\mathcal{G}$ is constrained to

$$
\partial \mathcal{F}=\mathcal{D}_{T} \mathcal{G}
$$

by the zero curvature condition. Thus, the Calabi flow is indeed an integrable twodimensional system of Toda type.

Next, the structure of the algebra (5.7) is analyzed in more detail, using components in $R^{1 \mid 1}$ superspace, and then used further to integrate the equation by group theoretical methods, as in ordinary Toda systems.

\subsection{Working in components}

The algebra introduced for the Calabi flow is not a supersymmetric extension of what was used before for the Ricci flow, but it is bosonic. Our prescription did not involve any substitution of the space derivatives $\partial$ and $\bar{\partial}$ by the supersymmetric covariant derivatives $\mathcal{D}_{ \pm}$, respectively, as it is customary in supersymmetric systems, but only used the odd 
generalization of the time evolution operator in $R^{1 \mid 1}$ superspace, in order to converge a second order differential equation in $(z, \bar{z})$ into fourth order. It can be alternatively viewed as changing the kernels of the algebra for the Ricci flow,

$$
S\left(t, t^{\prime}\right)=\delta\left(t-t^{\prime}\right), \quad K\left(t, t^{\prime}\right)=-\partial_{t} \delta\left(t-t^{\prime}\right),
$$

into the two super-kernels, respectively,

$$
\begin{aligned}
& S\left(T, T^{\prime}\right)=\delta\left(T-T^{\prime}\right)=\left(\theta-\theta^{\prime}\right) \delta\left(t-t^{\prime}\right), \\
& K\left(T, T^{\prime}\right)=-\mathcal{D}_{T} \delta\left(T-T^{\prime}\right)=-\delta\left(t-t^{\prime}\right)-\theta \theta^{\prime} \partial_{t} \delta\left(t-t^{\prime}\right),
\end{aligned}
$$

thus giving rise to the bosonic supercontinual Lie algebra with $K=\mathcal{D}_{T}$. Of course, the passage from smeared to unsmeared form of the algebra has to be done with care, since the integration over the Grassmann coordinates $\theta$ and $\theta^{\prime}$ will flip signs in some places. This generalization also changes the symmetry of the corresponding kernels, since $S\left(T, T^{\prime}\right)$ is now anti-symmetric and $K\left(T, T^{\prime}\right)$ is symmetric under the interchange of their arguments, $T \leftrightarrow T^{\prime}$.

It is useful in many respects to work out the form of the algebra in components by expanding the generators,

$$
X^{ \pm}(T)=X_{0}^{ \pm}(t)+\theta X_{1}^{ \pm}(t), \quad H(T)=H_{0}(t)+\theta H_{1}(t) .
$$

All components $X_{i}^{ \pm}(t)$ and $H_{i}(t)$ with $i=0,1$ are bosonic, and one arrives to the equivalent description of the local part of the algebra with commutation relations

$$
\begin{aligned}
& {\left[H_{0}(t), X_{0}^{ \pm}\left(t^{\prime}\right)\right]= \pm \delta\left(t-t^{\prime}\right) X_{0}^{ \pm}\left(t^{\prime}\right), \quad\left[X_{1}^{ \pm}(t), X_{0}^{\mp}\left(t^{\prime}\right)\right]= \pm \delta\left(t-t^{\prime}\right) H_{0}\left(t^{\prime}\right),} \\
& {\left[H_{0}(t), X_{1}^{ \pm}\left(t^{\prime}\right)\right]= \pm \delta\left(t-t^{\prime}\right) X_{1}^{ \pm}\left(t^{\prime}\right), \quad\left[X_{1}^{+}(t), X_{1}^{-}\left(t^{\prime}\right)\right]=\delta\left(t-t^{\prime}\right) H_{1}\left(t^{\prime}\right),} \\
& {\left[H_{1}(t), X_{1}^{ \pm}\left(t^{\prime}\right)\right]= \pm \partial_{t} \delta\left(t-t^{\prime}\right) X_{0}^{ \pm}\left(t^{\prime}\right),}
\end{aligned}
$$

whereas the remaining ones are trivial, i.e.,

$$
\begin{aligned}
& {\left[H_{1}(t), X_{0}^{ \pm}\left(t^{\prime}\right)\right]=0, \quad\left[X_{0}^{+}(t), X_{0}^{-}\left(t^{\prime}\right)\right]=0,} \\
& {\left[H_{i}(t), H_{j}\left(t^{\prime}\right)\right]=0 .}
\end{aligned}
$$

Smearing with ordinary functions of the continuous variable $t$, the algebra takes the form

$$
\begin{array}{ll}
{\left[H_{0}(\varphi), X_{0}^{ \pm}(\psi)\right]= \pm X_{0}^{ \pm}(\varphi \psi),} & {\left[X_{1}^{ \pm}(\varphi), X_{0}^{\mp}(\psi)\right]= \pm H_{0}(\varphi \psi),} \\
{\left[H_{0}(\varphi), X_{1}^{ \pm}(\psi)\right]= \pm X_{1}^{ \pm}(\varphi \psi),} & {\left[X_{1}^{+}(\varphi), X_{1}^{-}(\psi)\right]=H_{1}(\varphi \psi),} \\
{\left[H_{1}(\varphi), X_{1}^{ \pm}(\psi)\right]=\mp X_{0}^{ \pm}\left(\varphi^{\prime} \psi\right),} &
\end{array}
$$

and

$$
\begin{aligned}
& {\left[H_{1}(\varphi), X_{0}^{ \pm}(\psi)\right]=0, \quad\left[X_{0}^{+}(\varphi), X_{0}^{-}(\psi)\right]=0,} \\
& {\left[H_{i}(\varphi), H_{j}(\psi)\right]=0 .}
\end{aligned}
$$


One could have started directly from such an algebra, without prior justification, as in reference [24], and use it to establish the integrability of the Calabi flow ${ }^{2}$. However, employing the notion of supercontinual Lie algebras proves useful for putting its structure in neat form and making direct connection with the special class of integrable systems of Toda type.

The zero curvature formulation of the Calabi flow based on the algebra (5.7) can also be analyzed in components. The gauge connections (5.9) take the form

$$
A_{+}=H_{0}(f)+H_{1}(g)+X_{1}^{+}(1), \quad A_{-}=X_{0}^{-}\left(\Psi e^{\Phi}\right)+X_{1}^{-}\left(e^{\Phi}\right),
$$

using the expansion of the mixed superfields $\mathcal{F}=\Phi+\theta \Psi$ and $\mathcal{G}=g+\theta f$. Also, the relation (5.10) among these fields reads in components as

$$
\partial \Phi=f, \quad \partial \Psi=-\partial_{t} g .
$$

As a result, the zero curvature condition implies two more equations that follow by comparing coefficients of the terms $H_{0}$ and $H_{1}$, respectively,

$$
\bar{\partial} f=\Psi e^{\Phi}, \quad \bar{\partial} g=e^{\Phi},
$$

and lead to the system (5.6) that accounts for the Calabi flow, after eliminating all other functions but $\Phi$.

\subsection{Integration of Calabi flow}

The general solution can be obtained in analogy to the Ricci flow equation, as in ordinary Toda systems, by introducing a normalized highest weight state $|T\rangle$, assuming that it exists, which depends on the continuous coordinate $T$ of $R^{1 \mid 1}$ superspace with $\langle T| T>=$ 1. Equivalently, in terms of components $(t, \theta)$, we may assume that the highest weight state is expanded as

$$
|T>=| t>_{0}+\theta \mid t>_{1}
$$

with the orthonormalization conditions that the bosonic state $\mid t>_{0}$ has unit norm and it is perpendicular to $\mid t>_{1}$. It is more convenient to present its defining properties in smeared form ${ }^{3}$,

$$
X^{+}(\mathcal{F})|\mathcal{G}>=0, \quad<\mathcal{G}| X^{-}(\mathcal{F})=0, \quad H(\mathcal{F})|\mathcal{G}>=| \mathcal{F} \mathcal{G}>
$$

\footnotetext{
${ }^{2}$ There is a slight difference of signs in the commutation relations of the algebra and those appearing in reference [24]. The two choices are simply related to each other by changing $X_{0}^{ \pm} \rightarrow i X_{0}^{ \pm}$and $X_{1}^{ \pm} \rightarrow \mp i X_{1}^{ \pm}$and they do not affect the proof of integrability. The signs that arise here guarantee that the algebra is compatible with the Hermiticity condition $H_{i}^{\dagger}=H_{i}$ and $X_{i}^{ \pm}{ }^{\dagger}=X_{i}^{\mp}$ for all $i=0,1$, unlike the other case. These Hermiticity conditions turn out to be essential for the actual integration of the equation by group theoretical methods using highest weight representations, as will be seen later.

${ }^{3}$ Note that we systematically avoid to write the commutation relations of the supercontinual Lie algebra and the definition of its highest weight state starting from the unsmeared form of the supergenerators $A(T)$ labeled by $T$ in $R^{1 \mid 1}$. In this way, various sign discrepancies associated to the ambiguity of ordering products of super-functions defined at different points of super-space are avoided, as they can easily lead to errors.
} 
where $A(\mathcal{F})=A_{0}\left(\varphi_{1}\right)+A_{1}\left(\varphi_{0}\right)$ for the generators of the algebra and

$$
\left|\mathcal{F}>=\int\right| T>\mathcal{F}(T) d T=\int\left(\varphi_{1}(t)\left|t>_{0}+\varphi_{0}(t)\right| t>_{1}\right) d t=\left|\varphi_{1}>_{0}+\right| \varphi_{0}>_{1}
$$

for the highest weight state, using the smearing super-function $\mathcal{F}(T)=\varphi_{0}(t)+\theta \varphi_{1}(t)$.

The components of equation (5.23) satisfy the smeared relations

$$
\begin{aligned}
& H_{0}(\varphi)\left|\psi>_{0}=0, \quad H_{0}(\varphi)\right| \psi>_{1}=\mid \varphi \psi>_{0}, \\
& H_{1}(\varphi)\left|\psi>_{i}=\right| \varphi \psi>_{i} \quad \text { for } i=0,1,
\end{aligned}
$$

and

$$
X_{0}^{+}(\varphi)\left|\psi>_{i}=0=X_{1}^{+}(\varphi)\right| \psi>_{i} \quad \text { for } i=0,1
$$

together with their Hermitian conjugate equations, using ordinary functions $\varphi(t)$ and $\psi(t)$. The same relations can also be written in unsmeared bosonic form, as

$$
\begin{aligned}
& H_{0}\left(t^{\prime}\right)\left|t>_{0}=0, \quad H_{0}\left(t^{\prime}\right)\right| t>_{1}=\delta\left(t-t^{\prime}\right) \mid t>_{0}, \\
& H_{1}\left(t^{\prime}\right)\left|t>_{i}=\delta\left(t-t^{\prime}\right)\right| t>_{i} \quad \text { for } i=0,1,
\end{aligned}
$$

and

$$
X_{0}^{+}\left(t^{\prime}\right)\left|t>_{i}=0=X_{1}^{+}\left(t^{\prime}\right)\right| t>_{i} \quad \text { for } i=0,1 .
$$

It follows immediately from above that $H_{0}\left(t^{\prime}\right)\left|T>=\theta \delta\left(t-t^{\prime}\right)\right| t>_{0}$ and $H_{1}\left(t^{\prime}\right) \mid T>=$ $\delta\left(t-t^{\prime}\right) \mid T>$, which will be useful later for the calculations. These relations also imply, in particular, the following condition for the unsmeared action of the Cartan generator on the highest weight state $H\left(T^{\prime}\right)\left|T>=\left(\theta+\theta^{\prime}\right) \delta\left(t-t^{\prime}\right)\right| T>$, which is neither $\delta\left(T-T^{\prime}\right) \mid T>$ nor $\mid T>\delta\left(T-T^{\prime}\right)$ as compared to naive expectation.

The zero curvature formulation of the Calabi flow, which is better stated as superevolution equation (5.3), allows us to express its general solution in superfield form

$$
\mathcal{F}(z, \bar{z} ; t, \theta)=\mathcal{F}_{0}(z, \bar{z} ; t)-\mathcal{D}_{T}\left(\log <T\left|M_{+}^{-1}(z) M_{-}(\bar{z})\right| T>\right)
$$

where $\mathcal{F}_{0}=\Phi_{0}+\theta \Psi_{0}$ is a one-parameter family of mixed superfields whose components are two-dimensional bosonic free fields that depend on the continuous variable $t$,

$$
\Phi_{0}(z, \bar{z} ; t)=f(z ; t)+\bar{f}(\bar{z} ; t), \quad \Psi_{0}(z, \bar{z} ; t)=\psi(z ; t)+\bar{\psi}(\bar{z} ; t)
$$

Also, setting $\mathcal{F}_{0}(z ; T)=f(z ; t)+\theta \psi(z ; t)$ and $\overline{\mathcal{F}}_{0}(\bar{z} ; t)=\bar{f}(\bar{z} ; t)+\theta \bar{\psi}(\bar{z} ; t)$, the operators $M_{ \pm}$are given by the following path-ordered exponentials

$$
\begin{aligned}
& M_{+}(z)=\mathcal{P} \exp \left(\int^{z} d z^{\prime} \int e^{\mathcal{F}_{0}\left(z^{\prime} ; T^{\prime}\right)} X^{+}\left(T^{\prime}\right) d T^{\prime}\right) \\
& M_{-}(\bar{z})=\mathcal{P} \exp \left(\int^{\bar{z}} d \bar{z}^{\prime} \int e^{\overline{\mathcal{F}}_{0}\left(\bar{z}^{\prime} ; T^{\prime}\right)} X^{-}\left(T^{\prime}\right) d T^{\prime}\right) .
\end{aligned}
$$


Performing the integration over the anti-commuting variable $\theta^{\prime}$ that enters in the exponent, one arrives at the equivalent expressions in terms of components, respectively,

$$
\begin{aligned}
& M_{+}(z)=\mathcal{P} \exp \left(\int^{z} d z^{\prime} \int d t^{\prime} e^{f\left(z^{\prime} ; t^{\prime}\right)}\left[X_{1}^{+}\left(t^{\prime}\right)+\psi\left(z^{\prime} ; t^{\prime}\right) X_{0}^{+}\left(t^{\prime}\right)\right]\right), \\
& M_{-}(\bar{z})=\mathcal{P} \exp \left(\int^{\bar{z}} d \bar{z}^{\prime} \int d t^{\prime} e^{\bar{f}\left(\bar{z}^{\prime}, t^{\prime}\right)}\left[X_{1}^{-}\left(t^{\prime}\right)+\bar{\psi}\left(\bar{z}^{\prime} ; t^{\prime}\right) X_{0}^{-}\left(t^{\prime}\right)\right]\right) .
\end{aligned}
$$

Then, formula (5.29) summarizes the Bäcklund transformation of the Calabi flow, as a continual Toda system, and provides realization of its general solution for the arbitrary free field configuration $\left(\Phi_{0}, \Psi_{0}\right)$. The solution for $\Phi(z, \bar{z} ; t)$ is obtained by comparing the even terms of the mixed superfield equation (5.29), whereas $\Psi(z, \bar{z} ; t)$ is obtained from the odd terms; the resulting expressions, which are only implicitly defined here, should satisfy the equation $\Psi=\exp (-\Phi) \partial \bar{\partial} \Phi$, by construction. The (formal) power series expansion of the solution follows by expanding the path-ordered exponentials, as usual.

In the following we will consider first, for simplicity, the class of solutions with $\Psi_{0}(z, \bar{z} ; t)=0$, as they also resemble the solutions of the Ricci flow equation in some respect. The possibility to include free field configurations with $\Psi_{0} \neq 0$ will be considered separately, at the end of this section, as they lead to more complicated expressions for the free field expansion of the general solution.

(i) Solutions with $\Psi_{0}=0$ : In this case, the path-ordered exponentials (5.32) simplify considerably, as they involve only the generators $X_{1}^{ \pm}\left(t^{\prime}\right)$ in their exponent. Then, the detailed structure of the solution can be worked out in power series, which assumes the special form

$$
\begin{aligned}
&<T \mid M_{+}^{-1} M_{-} \mid T>=1+\sum_{n=1}^{\infty}(-1)^{n} \int^{z} d z_{1} \cdots \int^{z_{n-1}} d z_{n} \int^{\bar{z}} d \bar{z}_{1} \cdots \int^{\bar{z}_{n-1}} d \bar{z}_{n} \times \\
& \times \int \prod_{i=1}^{n} d t_{i} \int \prod_{i=1}^{n} d t_{i}^{\prime} \exp f\left(z_{i} ; t_{i}\right) \exp \bar{f}\left(\bar{z}_{i} ; t_{i}^{\prime}\right) D_{T}^{\left\{t_{1}, \cdots, t_{n} ; t_{1}^{\prime}, \cdots, t_{n}^{\prime}\right\}}
\end{aligned}
$$

with the usual path-ordering prescription $z \geq z_{1} \geq \cdots \geq z_{n-1} \geq z_{n}$, and likewise for the $\bar{z}$ 's. The structure of the corresponding algebra (5.7) is fully encoded into the elements

$$
D_{T}^{\left\{t_{1}, t_{2}, \cdots, t_{m} ; t_{1}^{\prime}, t_{2}^{\prime}, \cdots, t_{m}^{\prime}\right\}}=<T\left|X_{1}^{+}\left(t_{1}\right) X_{1}^{+}\left(t_{2}\right) \cdots X_{1}^{+}\left(t_{n}\right) X_{1}^{-}\left(t_{n}^{\prime}\right) \cdots X_{1}^{-}\left(t_{2}^{\prime}\right) X_{1}^{-}\left(t_{1}^{\prime}\right)\right| T>
$$

that only involve strings of the component operators $X_{1}^{ \pm}(t)$, for $\Psi_{0}=0$. Of course, even in this case, the other components of the algebra generators, $X_{0}^{ \pm}(t)$ as well as $H_{i}(t)$, will also appear in the process of evaluating the corresponding matrix elements by pushing $X^{+}$'s to the right and $X^{-}$'s to the left, as they follow from the commutation relations.

These elements can be computed recursively, as usual, starting from the defining relation $\langle T \mid T\rangle=1$. The result turns out to be

$$
\begin{aligned}
& D_{T}^{\left\{t_{1} ; t_{1}^{\prime}\right\}}=\delta\left(t-t_{1}\right) \delta\left(t_{1}-t_{1}^{\prime}\right), \\
& D_{T}^{\left\{t_{1}, t_{2} ; t_{1}^{\prime}, t_{2}^{\prime}\right\}}=\delta\left(t-t_{1}\right) \delta\left(t_{1}-t_{1}^{\prime}\right) \delta\left(t_{2}-t_{2}^{\prime}\right)\left(2 \delta\left(t-t_{2}\right)+\theta \partial_{t} \delta\left(t-t_{2}\right)\right),
\end{aligned}
$$




$$
\begin{aligned}
D_{T}^{\left\{t_{1}, t_{2}, t_{3} ; t_{1}^{\prime}, t_{2}^{\prime}, t_{3}^{\prime}\right\}}=\delta\left(t-t_{1}\right) \delta\left(t_{1}-t_{1}^{\prime}\right) \delta\left(t-t_{3}\right) \delta\left(t_{2}-t_{3}^{\prime}\right) \delta\left(t_{3}-t_{2}^{\prime}\right) \times \\
\times\left(2 \delta\left(t-t_{2}\right)+\theta \partial_{t} \delta\left(t-t_{2}\right)\right)+ \\
+\delta\left(t-t_{1}\right) \delta\left(t_{1}-t_{1}^{\prime}\right) \delta\left(t_{2}-t_{3}^{\prime}\right) \delta\left(t_{3}-t_{2}^{\prime}\right) \times \\
\quad \times\left(\delta\left(t-t_{3}\right)+\theta \partial_{t} \delta\left(t-t_{3}\right)\right)\left(2 \delta\left(t-t_{2}\right)+\theta \partial_{t} \delta\left(t-t_{2}\right)\right)+ \\
+\delta\left(t-t_{1}\right) \delta\left(t_{1}-t_{1}^{\prime}\right) \delta\left(t_{2}-t_{2}^{\prime}\right) \delta\left(t_{3}-t_{3}^{\prime}\right) \times \\
\quad \times\left(2 \delta\left(t-t_{2}\right)+\theta \partial_{t} \delta\left(t-t_{2}\right)\right)\left(\delta\left(t-t_{3}\right)+\theta \partial_{t} \delta\left(t-t_{3}\right)+\theta \partial_{t_{2}} \delta\left(t_{2}-t_{3}\right)\right)- \\
-\delta\left(t-t_{1}\right) \delta\left(t-t_{2}\right) \delta\left(t_{1}-t_{1}^{\prime}\right) \delta\left(t_{2}-t_{2}^{\prime}\right) \delta\left(t_{3}-t_{3}^{\prime}\right) \partial_{t} \delta\left(t-t_{3}\right)
\end{aligned}
$$

and so on. Higher order terms with $n>3$ are also calculable, but their expressions turn out to be quite lengthy with rapidly increasing complexity. For this reason they are not appended here, although contributions up to $n=4$ and 5 will be included in the subsequent evaluation of the field $\Phi$.

The expressions above should also be compared to the matrix elements (4.7) corresponding to the Ricci flow, as they show many similarities for small values of $n$. Observe that the time derivatives of delta functions appearing in the Ricci flow matrix elements now come multiplied with $-\theta$, which is consistent with the change of $\partial / \partial t$ to $\mathcal{D}_{T}=\partial / \partial \theta-\theta \partial / \partial t$ as one passes to the algebra of the Calabi flow. However, there is also a new term appearing in the last line of the $n=3$ matrix element, with no analogue in the Ricci flow, which contributes an additional term, without $\theta$, involving the operator $\mathcal{D}_{T}^{2}=-\partial / \partial t$ in the general power series expansion. The differences are becoming more visible in the structure of higher order terms, reflecting the more complicated nature of the commutation relations, which now involve a system of six rather than three basic bosonic generators of the algebra that all mix among themselves.

It is relatively easy to perform the path-ordered integrations and work out the form of the power series expansion for axially symmetric deformations that depend only on the combination $Y=z+\bar{z}$ for which the free field has the simpler looking form $\Phi_{0}(Y ; t)=$ $c Y+d(t)$, as for the Ricci flow. For constant values of the parameter $c$, it turns the element $<T\left|M_{+}^{-1} M_{-}\right| T>$ can be computed as series expansion in powers of $\exp \Phi_{0}$ and the Toda superfield $\mathcal{F}$ assumes the following form

$$
\begin{aligned}
\mathcal{F}= & \Phi_{0}+\frac{1}{(1 ! c)^{2}} \mathcal{D}_{T} e^{\Phi_{0}}+\frac{1}{\left(2 ! c^{2}\right)^{2}} \mathcal{D}_{T}\left(e^{\Phi_{0}} \mathcal{D}_{T} e^{\Phi_{0}}\right)+\frac{1}{\left(3 ! c^{3}\right)^{2}} \mathcal{D}_{T}\left(e^{2 \Phi_{0}} \mathcal{D}_{T}^{2} e^{\Phi_{0}}\right) \\
& +\frac{1}{\left(4 ! c^{4}\right)^{2}} \mathcal{D}_{T}\left(e^{3 \Phi_{0}} \mathcal{D}_{T}^{3} e^{\Phi_{0}}+11 e^{2 \Phi_{0}}\left(\mathcal{D}_{T} e^{\Phi_{0}}\right)\left(\mathcal{D}_{T}^{2} e^{\Phi_{0}}\right)\right) \\
& +\frac{1}{\left(5 ! c^{5}\right)^{2}} \mathcal{D}_{T}\left(e^{4 \Phi_{0}} \mathcal{D}_{T}^{4} e^{\Phi_{0}}+29 e^{3 \Phi_{0}}\left(\mathcal{D}_{T}^{2} e^{\Phi_{0}}\right)^{2}\right)+\cdots
\end{aligned}
$$

by taking into account the contribution of the matrix elements (5.34) up to order $n=5$. It is instructive to compare the terms with those appearing in the power series expansion (4.8) for axially symmetric deformations of the Ricci flow. To each order we see that the 
terms transform from one into the other by simply changing $\partial / \partial t$ into $\mathcal{D}_{T}$. Polynomial terms of the form $\left(\partial_{t} \exp \Phi_{0}\right)^{2}$, and alike, are missing from the Calabi flow, since they will correspond to $\left(\mathcal{D}_{T} \exp \Phi_{0}\right)^{2}$ and vanish for ordinary bosonic fields $\Phi_{0}$, due to $\theta^{2}=0$. As for the remaining terms, there are some differences in their relative coefficients that appear to order $n \geq 4$ in the expansion and reflect the differences of the two algebras, as expected on general grounds.

Substituting the result into the general expression (5.29) with $\Psi_{0}=0$ and comparing the coefficients of the even and odd terms, the expansion of $\Phi(Y ; t)$ in terms of free fields reads

$$
\Phi=\Phi_{0}-\frac{1}{\left(2 ! c^{2}\right)^{2}} e^{\Phi_{0}} \partial_{t} e^{\Phi_{0}}+\frac{e^{2 \Phi_{0}}}{\left(4 ! c^{4}\right)^{2}}\left(e^{\Phi_{0}} \partial_{t}^{2} e^{\Phi_{0}}+11\left(\partial_{t} e^{\Phi_{0}}\right)^{2}\right)+\cdots
$$

whereas the expansion for $\Psi(Y ; t)$ reads, up to fifth order,

$$
\begin{aligned}
\Psi= & e^{-\Phi} \partial_{Y}^{2} \Phi=-\frac{1}{(1 ! c)^{2}} \partial_{t} e^{\Phi_{0}}+\frac{e^{\Phi_{0}}}{\left(3 ! c^{3}\right)^{2}}\left(e^{\Phi_{0}} \partial_{t}^{2} e^{\Phi_{0}}+2\left(\partial_{t} e^{\Phi_{0}}\right)^{2}\right)- \\
& -\frac{e^{2 \Phi_{0}}}{\left(5 ! c^{5}\right)^{2}}\left(e^{2 \Phi_{0}} \partial_{t}^{3} e^{\Phi_{0}}+62 e^{\Phi_{0}}\left(\partial_{t} e^{\Phi_{0}}\right)\left(\partial_{t}^{2} e^{\Phi_{0}}\right)+87\left(\partial_{t} e^{\Phi_{0}}\right)^{3}\right)+\cdots
\end{aligned}
$$

The validity of these expressions can also be verified directly, order by order, by substituting into the system (5.6) that describes the Calabi flow equation. However, unlike the Ricci flow, there are no simple mini-superspace solutions that describe axially symmetric deformations of the round sphere by the Calabi flow in closed form and which resemble the sausage model. It remains an interesting open question to find such models, provided that there are consistent mini-superspace reductions of the Calabi flow, and compare the exact solutions with the formal power series expansion above. Similar remarks also apply to solutions with non-trivial free field component $\Psi_{0}$ that are examined next.

(ii) Solutions with $\Psi_{0} \neq 0$ : In this case, the choice of fields should be compatible with the equations

$$
e^{\Phi_{0}} \Psi_{0}=\partial \bar{\partial} \Phi_{0}, \quad \partial \bar{\partial} \Psi_{0}=-\frac{\partial e^{\Phi_{0}}}{\partial t}
$$

when applied to the most general free field configuration $\left(\Phi_{0}, \Psi_{0}\right)$. Of course, both sides of these equations vanish, since $\Phi_{0}$ is a two-dimensional free field that approximates well $\Phi$ only in a region of space where $\exp \Phi_{0} \simeq 0$, thus leaving $\Psi_{0}$ undetermined. Such asymptotic behavior is commonly assumed in all theories of Toda type in order for the formal power series expansion of their solutions to be meaningful, if also convergent. A simple choice is to consider $\Psi_{0}$ independent of the coordinates $(z, \bar{z})$, as it will provide free field expansion for the configurations of Calabi flow around constant curvature spheres, with $R=-\Psi_{0}$, and in the vicinity of those points where $\Phi_{0} \rightarrow-\infty$. The choice $\Psi_{0}=0$ that has been considered so far corresponds to such spheres, but with infinite radius, whereas in general one may assume that $\Psi_{0}$ is non-zero, but it can also depend on time, $\Psi_{0}(t)$. Even more generally, $\Psi_{0}$ can be an arbitrary family of two-dimensional free fields that are independent of the choice $\Phi_{0}$. The analysis below is quite general, without 
assuming any restrictions on the form of $\Psi_{0}$, apart from the case of axially symmetric solutions that are only considered for illustrative purposes. Other interesting possibilities also arise by specializing to particular classes of free field configurations, as will be seen later.

The calculations are considerably more involved now for evaluating the expectation value $<T\left|M_{+}^{-1}(z) M_{-}(\bar{z})\right| T>$ term by term in the power series expansion. Already, the first non-trivial term arising at order $n=1$ takes the form

$$
\begin{aligned}
\int^{z} d z_{1}^{\prime} \int^{\bar{z}} d \bar{z}_{1}^{\prime} \int d t_{1} \int d t_{1}^{\prime} \exp f\left(z_{1}^{\prime} ; t_{1}\right) \exp \bar{f}\left(\bar{z}_{1}^{\prime} ; t_{1}^{\prime}\right) \times \\
\quad \times<T\left|\left[X_{1}^{+}\left(t_{1}\right)+\psi\left(z_{1}^{\prime} ; t_{1}\right) X_{0}^{+}\left(t_{1}\right)\right]\left[X_{1}^{-}\left(t_{1}^{\prime}\right)+\bar{\psi}\left(\bar{z}_{1}^{\prime} ; t_{1}^{\prime}\right) X_{0}^{-}\left(t_{1}^{\prime}\right)\right]\right| T>(5
\end{aligned}
$$

as can be readily seen using the path-ordered exponentials (5.32) for $M_{ \pm}$. For $\Psi_{0} \neq 0$, there is an additional contribution coming from the cross terms in the expectation value, which were not present before. For higher values of $n$ there are many more new terms that contribute to the final answer, making the process tedious.

As before, it is also convenient here to restrict attention to axially symmetric configurations that depend only on the variable $Y=z+\bar{z}$ and assume that the two independent free fields are parametrized as follows,

$$
\Phi_{0}=c(z+\bar{z})+d(t), \quad \Psi_{0}=a(z+\bar{z})+b(t)
$$

where $a$ and $c$ are arbitrary constants whereas $b(t)$ and $d(t)$ depend generically on time. The path-ordered integrals can be evaluated easily in this case, but the resulting expressions are more lengthy than the simpler case with $\Psi_{0}=0$. It is quite instructive, nevertheless, to present the contributions to order $n=1$ and 2 ,

$$
\begin{aligned}
&<T \mid M_{+}^{-1} M_{-} \mid T>=1-\frac{1}{(1 ! c)^{2}} e^{\Phi_{0}}\left(1+\theta\left(\Psi_{0}-\frac{2 a}{c}\right)\right)+\frac{1}{\left(2 ! c^{2}\right)^{2}}\left[\theta e^{\Phi_{0}} \partial_{t} e^{\Phi_{0}}\right. \\
&\left.-\left(\Psi_{0}-\frac{3 a}{c}-2\right) e^{2 \Phi_{0}}-\theta\left(\Psi_{0}^{2}-4\left(1+\frac{a}{c}\right) \Psi_{0}+\frac{8 a}{c}+\frac{7 a^{2}}{2 c^{2}}\right) e^{2 \Phi_{0}}\right]
\end{aligned}
$$

whereas higher terms are of order $\exp \left(3 \Phi_{0}\right)$ or more.

Then, plugging into the general expression (5.29) and comparing the even and odd parts of the equation, it follows to this oder that

$$
\Phi=\Phi_{0}+\frac{1}{(1 ! c)^{2}}\left(\Psi_{0}-\frac{2 a}{c}\right) e^{\Phi_{0}}-\frac{1}{\left(2 ! c^{2}\right)^{2}}\left(e^{\Phi_{0}} \partial_{t} e^{\Phi_{0}}-\left(\Psi_{0}^{2}-\frac{4 a}{c} \Psi_{0}+\frac{7 a^{2}}{2 c^{2}}\right) e^{2 \Phi_{0}}\right)
$$

and

$$
\Psi=\Psi_{0}-\frac{1}{(1 ! c)^{2}} \partial_{t} e^{\Phi_{0}}-\frac{1}{\left(2 ! c^{2}\right)^{2}} \partial_{t}\left(\left(\Psi_{0}-\frac{3 a}{c}\right) e^{2 \Phi_{0}}\right)
$$

It can be verified, as independent check, that these expansions are consistent, order by order in powers of $\exp \Phi_{0}$, with the dimensionally reduced equations $\partial_{Y}^{2} \Phi=\Psi \exp \Phi$ and $\partial \Psi=-\partial_{t} \exp \Phi$ accounting for axially symmetric deformations along the Calabi flow. Higher order corrections can also be included, if required. 
Finally, note in passing that there are special configurations in which both free fields $\Phi_{0}$ and $\Psi_{0}$ do not depend on time, $t$, and which can be used to provide power series solutions of the simpler equation $\Delta \Delta \Phi=0$; equivalently they can be thought as describing Bäcklund transformations of the non-linear equation $\partial \bar{\partial} \Phi=\Psi_{0} \exp \Phi$, which looks like a generalized Liouville theory with space dependent coupling $\Psi_{0}$. Our method is also capable to provide solutions of this equation in terms of an arbitrary free field $\Phi_{0}$ for any given field $\Psi_{0}$. We will say more about this time independent class of configurations, and their formal power series solutions, while discussing the so called type III Robinson-Trautman metrics in four dimensional general relativity.

\section{Robinson-Trautman space-times}

In this section we discuss the embedding of the two-dimensional Calabi flow into fourdimensional vacuum Einstein equations and review the main characteristics of the spacetime solutions that are known up to this day. This material is included to make the presentation self-contained, as it allows to put our results in the physical context of spherical gravitational waves.

\subsection{General structure of the metrics}

Recall that the most general gravitational vacuum solution in four space-time dimensions which admits a geodesic, shear-free, twist-free but diverging null congruence is given by the general class of Robinson-Trautman metrics, [25]

$$
d s^{2}=2 r^{2} e^{\Phi} d z d \bar{z}-2 d t d r-H d t^{2}
$$

where $H(z, \bar{z}, r, t)$ has the special form

$$
H=r \partial_{t} \Phi-\Delta \Phi-\frac{2 m(t)}{r}
$$

expressed in terms of a single function $\Phi(z, \bar{z} ; t)$, and $m(t)$ is a one-parameter family of parameters that in some cases represent the physical mass of the system. The affine variable $r$ varies along the rays of the repeated null eigenvector and $t$ is a retarded time coordinate. Closed surfaces with constant $r$ and $t$ represent distorted two-dimensional spheres, and, therefore, $t$-dependent solutions are thought to describe spherical gravitational radiation; of course, these are not exactly spherical gravitational waves in vacuum, since spherical symmetry implies that the above metric is static. It is more appropriate to think of the Robinson-Trautman metrics as describing gravitational radiation outside some bounded region.

There is a close analogy between Lienard-Wiechert fields of Maxwell equations and Robinson-Trautman metrics of Einstein equations in that they both admit a principal null vector field which is geodesic, shear and twist free with non-vanishing divergence, 
thus giving a more intuitive meaning to the general ansatz that was made above, [26]. In the gravitational case, the field equations imply that all vacuum solutions in this class satisfy the following fourth-order differential equation for $\Phi(z, \bar{z} ; t)$,

$$
\Delta \Delta \Phi+3 m \partial_{t} \Phi+2 \partial_{t} m(t)=0
$$

where $\Delta=\exp (-\Phi) \partial \bar{\partial}$ as before. To understand its equivalence to the Calabi flow in two dimensions, (5.1), it suffices to relabel the null hypersurfaces of the Robinson-Trautman metrics and set $m$ equal to constant whenever $m(t) \neq 0$. The physically interesting case corresponds to positive values of $m$, in which case $t$ runs forward to infinity, but one may also consider the opposite case as well. Then, in the gauge with constant $m$, the Robinson-Trautman equation becomes

$$
\Delta \Delta \Phi+3 m \partial_{t} \Phi=0
$$

and it is identified with the Calabi flow by simple rescaling of the retarded time, so that $3 m=1$; as such it provides an exact embedding of the two-dimensional Calabi flow into four-dimensional gravity, as it was first noted in reference [27].

Finally, we note for completeness that there are also interesting generalizations of the present framework to Robinson-Trautman space-times with cosmological constant $\Lambda$, which are obtained by modifying the function (6.2) appearing in the metric to $H_{\Lambda}=$ $H-\Lambda r^{2} / 3$. Vacuum Einstein equations with cosmological constant reduce again to the same differential equation, as given in (6.3), which turns out to be independent of $\Lambda$. For an overview of the subject, see, for instance, [28].

\subsection{Petrov classification of metrics}

Next, following [28], we review the Petrov classification of Robinson-Trautman vacuum solutions depending on the algebraic relations among the surviving components of the Weyl curvature tensor that are typically represented by the quantities

$$
\begin{aligned}
& \Psi_{2}=-\frac{m}{r^{3}}, \quad \Psi_{3}=\frac{1}{2 r^{2}} e^{-\Phi / 2} \bar{\partial}(\Delta \Phi), \\
& \Psi_{4}=\frac{1}{2 r^{2}} \bar{\partial}\left(e^{-\Phi} \bar{\partial}\left(r \partial_{t} \Phi-\Delta \Phi\right)\right)
\end{aligned}
$$

and their complex conjugate expressions. There are four different algebraic types of space-times according to the list

$$
\begin{array}{ll}
\text { Type N : } & \Psi_{2}=\Psi_{3}=0, \\
\text { Type III : } & \Psi_{2}=0, \quad \Psi_{3} \neq 0, \\
\text { Type D : } & 3 \Psi_{2} \Psi_{4}=2 \Psi_{3}{ }^{2}, \quad \Psi_{2} \neq 0, \\
\text { Type II : } & 3 \Psi_{2} \Psi_{4} \neq 2 \Psi_{3}{ }^{2}, \quad \Psi_{2} \neq 0 .
\end{array}
$$


The mass parameter $m$, which is taken constant, vanishes for the first two types and as a result the Robinson-Trautman equation reduces to

$$
\Delta \Delta \Phi=0,
$$

with $t$ appearing as spectator without apparent role in dynamics. The last two types are characterized by the condition $m \neq 0$ and they correspond to genuine trajectories of the Calabi flow as given by equation (6.4). More precisely, we have the following:

Type $N$ solutions satisfy additional conditions, by their definition, which give rise to the special equation

$$
\partial \bar{\partial} \Phi=K(t) e^{\Phi},
$$

using an arbitrary integration function of the variable $t$ alone, $K(t)$, that plays the role of coupling constant in the resulting two-dimensional Liouville equation. In this case, the $t$ dependence of space-times is not in general compatible with the Calabi flow, since $m=0$, unless $K(t)$ is constant. We only note here that flat space-time arises as special solution of this type, whereas non-flat type $N$ solutions typically have lines of singularities in the three-dimensional $(r, z, \bar{z})$ space and they are also of limited interest in general relativity.

Type III solutions satisfy the equation $\Delta \Delta \Phi=0$, as before, with the condition

$$
\bar{\partial}(\Delta \Phi) \neq 0
$$

and its complex conjugate, by their definition. Therefore, space-times of this type correspond to solutions of the differential equation

$$
\Delta \Phi=f(z ; t)+\bar{f}(\bar{z} ; t),
$$

where $f$ and $\bar{f}$ are complex conjugate functions of $z$ and $\bar{z}$, respectively. By reparametrization of the complex coordinates one may choose without lose of generality functions $f(z)=z$ and $\bar{f}(\bar{z})=\bar{z}$ so that their characteristic equation becomes $\Delta \Phi=z+\bar{z}$. A particular simple solution is, [25],

$$
e^{\Phi}=\frac{3}{(z+\bar{z})^{3}} .
$$

More general static solutions of type III are hard to construct beyond the ones that can be obtained from (6.11) by arbitrary reparametrization of the coordinates $(z, \bar{z})$, whereas any dependence on $t$ is dropped out for all practical purposes.

Type D solutions are fully known and they include the Schwartzchild metric with mass parameter $m$ as static example. In this class, there are additional relations

$$
\bar{\partial}\left(e^{-\Phi} \bar{\partial}(\Delta \Phi)\right)=0, \quad e^{-\Phi}(\bar{\partial}(\Delta \Phi))^{2}+3 m \bar{\partial}\left(e^{-\Phi} \bar{\partial}\left(\partial_{t} \Phi\right)\right)=0,
$$

and their complex conjugate, which are obtained from the condition $3 \Psi_{2} \Psi_{4}=2 \Psi_{3}{ }^{2}$ to different orders in the radial variable $r$. Integration of the first equation yields 
$\exp (-\Phi) \bar{\partial}(\Delta \Phi)=f(z ; t)$, where $f(z)$ is an arbitrary analytic function. Then, the Robinson-Trautman equation (6.4) simplifies to

$$
\partial\left(f e^{\Phi}\right)+3 m \partial_{t} e^{\Phi}=0
$$

plus its complex conjugate relation.

For $f=0$ the corresponding metric is $t$-independent and takes the form

$$
d s^{2}=\frac{2 r^{2}}{\left(1+\frac{K}{2} z \bar{z}\right)^{2}} d z d \bar{z}-2 d t d r-\left(K-\frac{2 m}{r}\right) d t^{2}
$$

with constant $K$ that can be normalized to 0 or \pm 1 . For $K=1$ it coincides with the Schwartzschild metric in Eddington-Finkelstein frame, setting

$$
t=t_{s}-r-2 m \log (r-2 m)
$$

in terms of the usual time coordinate $t_{s}$ of the Schwartzschild solution. When $f$ is not zero, one can perform coordinate transformation to make it constant, say $f=-3 m$, in which case the function $\Phi$ depends only on the combination $z+\bar{z}+t$. Then, the geometry can be brought into the static $C$-metric form by further change of the coordinates (see, for instance, [28]). A notable example of this kind corresponds to the choice, [29],

$$
e^{\Phi}=\frac{A}{(z+\bar{z}+t)^{3 / 2}}
$$

having $4 m A^{2}=1$.

Type II solutions provide the last but most general class of space-times that are directly related to time dependent solutions of the Calabi flow. They admit arbitrary initial data $\Phi\left(z, \bar{z} ; t_{0}\right)$ at some given time $t=t_{0}$, but unfortunately there are hardly any explicit solutions known to this day, apart from those obtained from type III configurations by allowing $m \neq 0$, [25], as, for instance, the particular solution (6.11). Solution generating techniques have been subsequently employed toward the construction of more general type II space-times, but the results are rather inconclusive in the literature; see, however, the recent work [30] on type II metrics which provides some explicit solutions that are not asymptotically flat. The main interest in type $I I$ solutions stems from the fact that both gravitational radiation and black hole formation can be studied simultaneously in exact terms in the vacuum. Thus, it is an outstanding problem in general relativity to explore the general structure of type II solutions; here come our results to make a decisive step in this direction.

\subsection{Further physical considerations}

It is clear from the discussion above that type III and type II Robinson-Trautman metrics provide the most general classes of gravitational metrics (6.1) in four dimensional spacetimes with parameter $m$ zero and non-zero, respectively, whereas type $\mathrm{N}$ and type $\mathrm{D}$ metrics follow from them as special limiting cases. 
Type II metrics provide a class of radiative space-times which tend to the Schwarzschild solution in the asymptotic future. Actually, approximate solutions of this kind were first examined in the literature on general relativity, [31], by perturbing the Schwarzschild metric so that the 2 -spheres $M$ defined at constant $r$ and $t$ represent distorted configurations in the multi-pole expansion of gravitational radiation. In particular, using the linearized approximation, as outlined in section 2.5, small perturbations associated to spherical harmonics with $l \geq 2$ were found to decay exponential fast by inducing quadrapole and higher order pole radiation, until the configuration eventually settles back to the round sphere as $t \rightarrow \infty$. It was subsequently found that all dynamical type II solutions evolving toward the Schwarzschild solution exhibit Lyapunov stability using the quadratic curvature functional $S(g)$, given by equation (2.16) for $M=S^{2}$, [32]. As was also shown in section $2, S(g)$ varies monotonically with time and, in this case, the critical point where it assumes its minimal value is the round sphere that corresponds to the Schwarzschild space-time in Eddington-Filkenstein frame (6.14). In this case, the notion of extremal Kähler metrics that was introduced on general grounds by Calabi reduces to the constant curvature metric on $S^{2}$ that characterizes the end point of the dynamical evolution. Finally, the (semi)-global existence and convergence of solutions with smooth initial data has been established in all generality, following arguments similar to the long time existence and convergence of solutions to the Ricci flow, thus establishing the universal limiting character of all asymptotically flat type II metrics, [12] (but see also $[27,33]$ for important earlier work on the subject).

The global structure of Robinson-Trautman space-times has been carefully analyzed and extensions across the null hypersurface at $t=\infty$ have been considered in analogy to the Kruskal extension of the $r>2 m$ Schwarzschild space-time. Two such solutions can be glued together along their Schwarzschild-like event horizon to form space-times that contain both a black and a white hole, as in the ordinary static case. However, the extension through the horizon is not smooth, in general, which implies that an observer living in this space can determine by local measurements whether or not he has crossed the horizon. These results were obtained by a detailed asymptotic analysis of the long time behavior of solutions to the Robinson-Trautman-Calabi equation, which upon transformation to Kruskal type coordinates were found to exhibit terms in the power series expansion with logarithmic coefficients, [34]. When the cosmological constant is not zero, the analysis of the solutions and their long time behavior essentially remains unaltered. However, the value of $\Lambda$ enters in the space-time interpretation of the solutions and their long time asymptotic convergence to the Schwarzschild solution with a cosmological constant, e.g., the Schwarzschild-de Sitter metric when $\Lambda>0$. An interesting feature of such generalization is that the continuation of the metric across the horizon can be made with a higher degree of smoothness when $\Lambda>0$, [35], as compared to the more standard case with vanishing cosmological constant.

Previous attempts in the literature to integrate the Robinson-Trautman equation for type II metrics include the application of prolongation methods, as in reference [36]. However, their results remain rather preliminary and inconclusive as they felt short of 
expectation compared to other two-dimensional integrable systems that can be more easily treated by such tools. There are also some works on series solutions of type II metrics, [37], but a systematic understanding of their origin seems to be lacking. Our main contribution to this subject, according to the previous sections, is the ability to cast the class of all type II metrics into zero curvature form on the two-dimensional space $(z, \bar{z})$ and construct systematically formal solutions based on free fields, via group theoretical methods. The only drawback of our construction is the inability to characterize the global structure of the resulting space-times, and their possible singularities, in terms of the free field data used in the Bäcklund transformations. Also, simple but explicit solutions of the equations are still unknown, as they may never exist simple models of spherical gravitational radiation in vacuum that only depend on finite number of moduli.

Type III metrics, on the other hand, require separate investigation as they do not correspond to genuine dynamical solutions of the Calabi flow. True, they can be promoted to type II solutions of a very special type by allowing $m \neq 0$, but they also remain largely out of reach apart from specific solutions, such as (6.11), and their descendants by coordinate transformations. The zero curvature formulation of type III metrics will be considered separately in the next section, where it is also explained how to extend the group theoretical methods of Toda theory in order to construct formal solutions in terms of free fields, as in the fully dynamical situation. We only note here that type III metrics are characterized by the non-vanishing component of the Weyl curvature tensor $\Psi_{3}$, which in turn implies that $\partial(\Delta \Phi)$ and $\bar{\partial}(\Delta \Phi)$ differ from zero. Thus, type III metrics do not correspond to extremal Kähler metrics on $S^{2}$, using the terminology introduced by Calabi, [6, 7]. On $S^{2}$ the extremal metrics are also constant curvature metrics with constant $\Delta \Phi$.

\section{The algebraic description of type III metrics}

A zero curvature formulation of the non-linear equation that characterizes type III Robinson-Trautman metrics,

$$
\Delta \Delta \Phi=0
$$

was given in reference [24] by taking suitable limit of the commutation relations of the algebra used for the Calabi flow. Here, we provide another zero curvature formulation of the same equation, which is not equivalent to that construction, but is more natural for applying the general framework of Toda field theories and their solution generating techniques as in the Calabi flow.

First, notice that the equation (7.1) can be written in superfield form as

$$
\partial \bar{\partial} \mathcal{F}=\frac{\partial}{\partial \theta} e^{\mathcal{F}}
$$

using $\mathcal{F}=\Phi+\theta \Psi$ with bosonic components that do not not depend on time, i.e., $t$ will be spectator if added by hand. Comparing the even and odd parts of the equation it 
follows that

$$
\partial \bar{\partial} \Phi=\Psi e^{\Phi}, \quad \partial \bar{\partial} \Psi=0,
$$

which is equivalent to equation (7.1) after eliminating the field $\Psi$. Thus, equation (7.1) admits a formal Toda field theory description with Cartan operator $\partial / \partial \theta$.

Next, to appreciate the idea behind the new formulation of this equation that will be adopted in the sequel, let us briefly recall the essential features of Toda field equations based on the generalized system of commutation relations

$$
\begin{aligned}
& {\left[X^{+}(\mathcal{F}), X^{-}(\mathcal{G})\right]=H(S(\mathcal{F} \mathcal{G})), \quad[H(\mathcal{F}), H(\mathcal{G})]=0} \\
& {\left[H(\mathcal{F}), X^{ \pm}(\mathcal{G})\right]= \pm X^{ \pm}((K \mathcal{F}) \mathcal{G})}
\end{aligned}
$$

Lie algebras of this type provide a natural supercontinual extension of the more abstract class of continual Lie algebras (3.3) for any given pair of linear super-operators $(K, S)$. Of course, as in the ordinary bosonic case considered in section 3, a supercontinual Lie algebra with characteristics $(K, S)$ is isomorphic to that with $(\tilde{K}=K S, \tilde{S}=1)$, provided that the corresponding Cartan generators are related to each other by $\tilde{H}(\mathcal{F})=H(S \mathcal{F})$ when $S$ is invertible. Thus, one typically makes the canonical choice $(\tilde{K}, 1)$ without loss of generality.

The associated system of Toda field equations follows from the zero curvature condition $\left[\partial+A_{+}, \bar{\partial}+A_{-}\right]=0$ choosing the gauge connections

$$
A_{+}=H(\mathcal{G})+X^{+}(1), \quad A_{-}=X^{-}\left(e^{\mathcal{F}}\right) .
$$

The resulting equations read, in general,

$$
\partial \mathcal{F}=K \mathcal{G}, \quad \bar{\partial} \mathcal{G}=S e^{\mathcal{F}}
$$

and therefore one obtains

$$
\partial \bar{\partial} \mathcal{F}=K S e^{\mathcal{F}}=\tilde{K} e^{\mathcal{F}}
$$

after eliminating the field $\mathcal{G}$. Note that the final field equation only depends on the operator $\tilde{K}$ and as such it is inert to the choice of algebra $(K, S)$ or $(\tilde{K}, 1)$. However, the intermediate equations (7.6), which transform into one another when different choices are being made, will be equivalent only if $S$ is invertible operator.

Thus, it does not make a difference whether one uses $K=1$ and $S=\mathcal{D}_{T}$ or the canonical choice $K=\mathcal{D}_{T}$ and $S=1$, as we did before, for the zero curvature formulation of the Calabi flow. The situation changes drastically when the map $\tilde{H}(\mathcal{F})=H(S \mathcal{F})$ is degenerate, as in the case of nilpotent operators with $S^{2}=0$, for the two algebras with operators $(K, S)$ and $(\tilde{K}, 1)$ are not anymore isomorphic. This particular situation is realized when $S=\partial / \partial \theta$ and the algebra with characteristics $(\partial / \partial \theta, 1)$ is not isomorphic to that with $(1, \partial / \partial \theta)$. Although the associated Toda field equation, which in this case reads as (7.2), does not know the difference, the intermediate equations (7.6) are actually sensitive on the choice. Hence, the natural question arises which is the most recommended choice of algebra in the present case. 
Let us choose $K=1$ and $S=\partial / \partial \theta$ and examine the detailed form of the equations (7.6), which in terms of components $\mathcal{F}=\Phi+\theta \Psi$ and $\mathcal{G}=g+\theta f$ become, respectively,

$$
\begin{aligned}
& \partial \Phi=g, \\
& \bar{\partial} g=\Psi e^{\Phi}, \quad \bar{\partial} f=0 .
\end{aligned}
$$

Then, by eliminating $f$ and $g$ one arrives at the system (7.3) for the functions $\Phi$ and $\Psi$ that are required in all generality. On the other hand, the choice $K=\partial / \partial \theta$ and $S=1$ leads to a different system of intermediate equations (7.6), which in term of components read

$$
\begin{aligned}
& \partial \Phi=f, \quad \partial \Psi=0, \\
& \bar{\partial} g=e^{\Phi}, \quad \bar{\partial} f=\Psi e^{\Phi} .
\end{aligned}
$$

Eliminating $f$ and $g$, as before, we obtain the equations $\partial \bar{\partial} \Phi=\Psi e^{\Phi}$ and $\partial \Psi=0$, which also lead to $\Delta \Delta \Phi=0$ as required. However, the latter formulation is more restrictive than the first since $\partial \Psi=0$, and its complex conjugate equation $\bar{\partial} \Psi=0$, determine only a very special class of solutions to the second order equation $\partial \bar{\partial} \Phi=0$ appearing in the system (7.3). Thus, generality requires making the choice $(1, \partial / \partial \theta)$ rather than $(\partial / \partial \theta, 1)$. The different choices yield equivalent descriptions only for constant curvature metrics in two dimensions, in which case the fourth order differential equation (7.1) becomes equivalent to Liouville equation.

With these explanations in mind, we choose the supercontinual Lie algebra with characteristics $K=1$ and $S=\partial / \partial \theta$, whose commutation relations take the following form in terms of components, using the decomposition of the generators $A=A_{0}+\theta A_{1}$,

$$
\begin{array}{ll}
{\left[H_{1}(\varphi), X_{0}^{ \pm}(\psi)\right]= \pm X_{0}^{ \pm}(\varphi \psi),} & {\left[X_{1}^{ \pm}(\varphi), X_{0}^{\mp}(\psi)\right]= \pm H_{1}(\varphi \psi),} \\
{\left[H_{0}(\varphi), X_{1}^{ \pm}(\psi)\right]= \pm X_{0}^{ \pm}(\varphi \psi),} & {\left[H_{1}(\varphi), X_{1}^{ \pm}(\psi)\right]= \pm X_{1}^{ \pm}(\varphi \psi),}
\end{array}
$$

whereas the rest are trivial,

$$
\begin{array}{ll}
{\left[X_{0}^{+}(\varphi), X_{0}^{-}(\psi)\right]=0,} & {\left[X_{1}^{+}(\varphi), X_{1}^{-}(\psi)\right]=0,} \\
{\left[H_{0}(\varphi), X_{0}^{ \pm}(\psi)\right]=0,} & {\left[H_{i}(\varphi), H_{j}(\psi)\right]=0 .}
\end{array}
$$

These commutation relations should be contrasted with the choice made in reference [24], which is also quite general, but it constitutes an inequivalent algebraic description of the same problem which will not be utilized in the present work.

The formulation that is adopted here is more appropriate for integrating the equation $\Delta \Delta \Phi=0$ by group theoretical methods, at least formally, as in ordinary Toda systems. The present description has the advantage that $t$ is completely decoupled from the commutation relations of the algebra, and, as a result, the smearing functions in the components of the generators, $A_{0}(\varphi)$ and $A_{1}(\varphi)$, can be completely dropped out. Put differently, the smearing superfields $\mathcal{F}$ can be considered as functions of $\theta$ only, without 
having any $t$ dependence, so that they correspond to super-numbers $\varphi_{0}+\theta \varphi_{1}$. Also, the smeared generators can be simply defined by integration over the Grassmann variable $\theta$, as

$$
A(\mathcal{F})=\int A(\theta) \mathcal{F}(\theta) d \theta=\varphi_{1} A_{0}+\varphi_{0} A_{1}
$$

and, as a result, the $\varphi$ and $\psi$ dependence in the above commutation relations is superfluous and factors out completely.

Likewise, the highest weight state that is formally introduced to integrate the associated Toda field equation (7.2) assumes a simpler form, compared to the Calabi flow, as it can be taken independent of $t$. Thus, here, we consider a normalized vacuum state

$$
|\theta>=| 0>_{0}+\theta \mid 0>_{1}
$$

with defining relations, in smeared form,

$$
X^{+}(\mathcal{F})|\mathcal{G}>=0, \quad<\mathcal{G}| X^{-}(\mathcal{F})=0, \quad H(\mathcal{F})|\mathcal{G}>=| \mathcal{F G}>
$$

as before, where

$$
\left|\mathcal{F}>=\int\right| \theta>\mathcal{F}(\theta) d \theta=\varphi_{1}\left|0>_{0}+\varphi_{0}\right| 0>_{1}
$$

In terms of components these relations read

$$
\begin{array}{ll}
H_{0} \mid 0>_{0}=0, & H_{0}\left|0>_{1}=\right| 0>_{0}, \\
H_{1}\left|0>_{i}=\right| 0>_{i} & \text { for } i=0,1,
\end{array}
$$

and

$$
X_{0}^{+}\left|0>_{i}=0=X_{1}^{+}\right| 0>_{i} \quad \text { for } i=0,1
$$

together with their Hermitian conjugate relations.

Then, the group theoretical framework for Toda theories can be implemented in this case and the general solution of equation (7.2) takes the form

$$
\mathcal{F}(z, \bar{z} ; \theta)=\mathcal{F}_{0}(z, \bar{z} ; \theta)-\log <\theta\left|M_{+}^{-1}(z) M_{-}(\bar{z})\right| \theta>
$$

in accordance to the general expression (3.15) applied to supercontinual Toda systems with $K=1 . \mathcal{F}_{0}=\Phi_{0}+\theta \Psi_{0}$, whose components are two-dimensional free fields

$$
\Phi_{0}(z, \bar{z})=f(z)+\bar{f}(\bar{z}), \quad \Psi_{0}(z, \bar{z})=\psi(z)+\bar{\psi}(\bar{z})
$$

Finally, the operators $M_{ \pm}$are given by the path-ordered exponentials

$$
\begin{aligned}
& M_{+}(z)=\mathcal{P} \exp \left(\int^{z} d z^{\prime} e^{f\left(z^{\prime}\right)}\left[X_{1}^{+}+\psi\left(z^{\prime}\right) X_{0}^{+}\right]\right) \\
& M_{-}(\bar{z})=\mathcal{P} \exp \left(\int^{\bar{z}} d \bar{z}^{\prime} e^{\bar{f}\left(\bar{z}^{\prime}\right)}\left[X_{1}^{-}+\bar{\psi}\left(\bar{z}^{\prime}\right) X_{0}^{-}\right]\right)
\end{aligned}
$$

that do not involve integration over the continuous time variable $t$. 
Actually, there are several simplifications that occur in this case. Note that the commutation relations $(7.10)$ and $(7.11)$ contain a subalgebra generated by $X_{0}^{ \pm}, X_{1}^{ \pm}$ and $H_{1}$, which is sufficient to determine the structure of $\left\langle\theta\left|M_{+}^{-1} M_{-}\right| \theta\right\rangle$. The power series expansion of the path-ordered exponentials $M_{ \pm}$contain strings of these generators only, and, as a result, the evaluation of the corresponding vacuum expectation values, which is carried out as usual by pushing $X^{+}$'s to the right and $X^{-}$'s to the left, can never involve $H_{0}$ and its action on the highest weight state $\mid \theta>$. Since $H_{1}|\theta>=| \theta>$, as opposed to $H_{0}|\theta>=\theta| 0>_{0}$, it follows readily that the Grassmann variable $\theta$ never appears as coefficient in the resulting expressions, and, for all practical purposes, the vacuum expectation value can be restricted to the state $\mid 0>_{0}$ alone. Thus, $\theta$ is also a superfluous variable in writing the general form of the solution, which simplifies to

$$
\Phi(z, \bar{z})=\Phi_{0}(z, \bar{z})-\log \left({ }_{0}<0\left|M_{+}^{-1}(z) M_{-}(\bar{z})\right| 0>_{0}\right) .
$$

At the same time, by comparing the odd terms of equation (7.18), we obtain to all orders the result

$$
\Psi(z, \bar{z})=\Psi_{0}(z, \bar{z})
$$

thanks to the absence of $\theta$-dependent terms in the expression ${ }_{0}<0\left|M_{+}^{-1} M_{-}\right| 0>_{0}$.

By construction, the power series expansion of a general field configuration $\Phi$ in terms of free fields $\Phi_{0}$ should coincide with the expansion that governs the Calabi flow when restricted to $t$-independent fields, since this limit is well defined within the general class of Toda systems. Thus, up to second order in powers of $\exp \Phi_{0}$, it follows that for axially symmetric configurations with $\Psi_{0}=a(z+\bar{z})+b$ and $\Phi=c(z+\bar{z})+d$, a broad class of solutions is

$$
\Phi=\Phi_{0}+\frac{e^{\Phi_{0}}}{(1 ! c)^{2}}\left(\Psi_{0}-\frac{2 a}{c}\right)+\frac{e^{2 \Phi_{0}}}{\left(2 ! c^{2}\right)^{2}}\left(\Psi_{0}^{2}-\frac{4 a}{c} \Psi_{0}+\frac{7 a^{2}}{2 c^{2}}\right)+\cdots
$$

whereas $\Psi=\Psi_{0}$ to all orders in the expansion, as required. In the special case of constant curvature metrics, which correspond to $\Psi_{0}=b$, the system (7.3) reduces to the Liouville equation,

$$
\partial \bar{\partial} \Phi=b e^{\Phi}
$$

and axially symmetric solutions of it assume the free field expansion

$$
\Phi=\Phi_{0}+\frac{b}{(1 ! c)^{2}} e^{\Phi_{0}}+\frac{b^{2}}{\left(2 ! c^{2}\right)^{2}} e^{2 \Phi_{0}}+\cdots
$$

according to the more general expression given above.

Finally, it is interesting to identify the algebra whose local part is generated by $X_{0}^{ \pm}$, $X_{1}^{ \pm}$and $H_{1}$ after dropping the superficial dependence on the smearing functions. Using the commutation relations (7.10) and (7.11), and changing base to

$$
\begin{array}{ll}
e_{0}=\frac{1}{\sqrt{2}}\left(X_{0}^{+}+X_{1}^{+}\right), & e_{1}=\frac{1}{\sqrt{2}}\left(X_{0}^{+}-X_{1}^{+}\right), \\
f_{0}=\frac{1}{\sqrt{2}}\left(X_{0}^{-}+X_{1}^{-}\right), & f_{1}=\frac{1}{\sqrt{2}}\left(-X_{0}^{-}+X_{1}^{-}\right),
\end{array}
$$


it follows, setting also $H_{1}=h$, that

$$
\left[e_{i} f_{j}\right]=\delta_{i j} h, \quad\left[h, e_{i}\right]=e_{i}, \quad\left[h, f_{i}\right]=-f_{i} .
$$

These are precisely the defining relations of Kac's $K_{2}$ algebra, [38], which is the most elementary example of simple Lie algebra with infinite growth ${ }^{4}$. Equivalently, it can be thought as arising from the algebra

$$
\left[e_{i} f_{j}\right]=\delta_{i j} h_{j}, \quad\left[h_{i}, e_{j}\right]=K_{i j} e_{j}, \quad\left[h_{i}, f_{j}\right]=-K_{i j} f_{j}
$$

with $i$ and $j$ taking values 0 or 1 , using a degenerate $2 \times 2$ Cartan matrix with all its elements equal to 1 . In such case, $h_{0}-h_{1}$ is a central element, as it commutes with all other generators $e_{i}$ and $f_{i}$, and when it is factored out yields the $K_{2}$ algebra.

The $K_{2}$ algebra was encountered in the literature before as the prolongation structure of the equation $\Delta \Delta \Phi=0$ that characterizes type III Robinson-Trautman metrics, [39]. It is rewarding that it also arises here using a somewhat different route of investigation. Our general construction based on Toda theories also connects it nicely with the algebra that determines formal solutions of the Calabi flow. It should also be noted that there is yet another way to write the type III metrics in zero curvature form, following [24]. The algebra used there is more complicated, as it depends on the additional parameter $t$ thought as spectator - in a non-trivial way, and it only contains $K_{2}$ in its zero modes. The intertwining of these two different algebraic descriptions of the same equation deserves further attention as it may provide the means to construct non-trivial conservation laws. This problem is left open for future work.

\section{Hierarchy of higher order flows}

Motivated by the general relation between the Ricci and Calabi flows, we introduce a hierarchy of higher order geometric flows by taking successive square roots of the time derivative operator. We will first describe the construction in two dimensions and outline an algorithm for casting the corresponding equations into zero curvature form. Finally, we will discuss the generalization of the hierarchy of geometric evolution equations to Kähler manifolds of arbitrary dimension.

\subsection{Higher order flows in two dimensions}

Let us assume that the conformal factor of the metric in two dimensions depends on infinite many time variables, $t_{i}$, as

$$
\Phi=\Phi\left(z, \bar{z} ; t_{1}, t_{2}, t_{3}, t_{4}, \cdots\right)
$$

\footnotetext{
${ }^{4}$ The series of $K_{n}$ algebras is defined by the commutation relations (7.27) with $i$ and $j$ taking $n$ different values. In all cases there is only one Cartan generator $h$ and the growth of the algebras is infinite for $n \geq 2$. The case $n=1$ corresponds to the $\operatorname{sl}(2)$ algebra and naturally it is not included in this series.
} 
and that there is an associated hierarchy of intrinsic geometric flows,

$$
\frac{\partial \Phi}{\partial t_{n}}=\Psi_{n}(R)
$$

for appropriately chosen expressions of the scalar curvature $R=-\Delta \Phi$. The hierarchy is defined by imposing the following requirement for any two consecutive flows,

$$
\frac{\partial e^{\Phi}}{\partial t_{n+1}}=-\frac{\partial^{2} e^{\Phi}}{\partial t_{n}^{2}}
$$

This, in turn, implies $\partial_{t_{n+1}} \Phi=-\partial_{t_{n}}^{2} \Phi+\left(\partial_{t_{n}} \Phi\right)^{2}$, which suffices to determine recursively the form of curvature functionals $\Psi_{n}(R)$, starting from the Ricci flow that corresponds to the choice $\Psi_{1}=-R$ for the time variable $t_{1}$. We have, in particular,

$$
\Psi_{n+1}=-\frac{\partial \Psi_{n}}{\partial t_{n}}-\Psi_{n}^{2}
$$

which upon iteration yields $\Psi_{n}$ in terms of $R$. Also note that due to equation (8.3), the compatibility condition of the proposed hierarchy reads $\partial_{t_{n+1}}\left(\Psi_{n} \exp \Phi\right)=\partial_{t_{n}}\left(\Psi_{n+1} \exp \Phi\right)$. Thus, in the appropriate sense, when the time derivatives act upon $\exp \Phi$ rather than $\Phi$, the hierarchy (8.2) provides a system of independent flows; in this respect, it could be compared to other systems of non-linear differential equations with increasing order, such as the $\mathrm{KdV}$ hierarchy.

This procedure can be simply implemented as stated above. However, there is an alternative description in two dimensions that will allow to prove the integrability of these flows by uncovering the relevant algebraic structures, as was successfully done for the Calabi flow. In particular, let us start from $n$-th flow and make the substitution

$$
\frac{\partial}{\partial t_{n}} \rightarrow \frac{\partial}{\partial \theta_{n}}-\theta_{n} \frac{\partial}{\partial t_{n+1}}, \quad \Phi \rightarrow \Phi+\theta_{n}\left(\partial_{t_{n}} \Phi\right)
$$

with the aid of a Grassmann variable $\theta_{n}$, so that the $(n+1)$-flow is realized as superevolution partner of the $n$-th flow. As a result, the order of the resulting parabolic equation is doubled, so that the $n$-th member in the family of flows (8.2) has order $2^{n}$. The substitution made for $\Phi$ can be understood as requirement of its super-analyticity on the super-coordinate $T_{n}=\left(t_{n}, \theta_{n}\right)$ that extends $t_{n}$ in $R^{1 \mid 1}$ superspace; of course, $\partial_{t_{n}} \Phi=\Psi_{n}$, which in turn determines $\Psi_{n+1}$ by working out the form of the equation in components. Also, consistency of the scheme requires that the Grassmann variables $\theta_{i}$ introduced in each step are all independent, i.e., $\theta_{i} \theta_{j}+\theta_{j} \theta_{i}=2 \delta_{i j}$.

We have already seen in detail how this method yields the Calabi flow with respect to the time variable $t_{2}$ (in the present notation). Further iteration yields the following eighth order differential equation,

$$
\frac{\partial \Phi}{\partial t_{3}}=\Delta[(\Delta \Phi)(\Delta \Delta \Phi)-\Delta \Delta \Delta \Phi]=\Delta(R \Delta R+\Delta \Delta R)
$$


using the expression $\Psi_{2}=-\Delta \Delta \Phi$ for the Calabi flow. The integrability of this equation will be established later by implementing its algorithmic construction to the level of supercontinual Lie algebras.

The structure of the equations becomes considerably more involved for higher values of $n$, as their order increases exponentially. We only give here the next one, for illustration,

$$
\begin{aligned}
\frac{\partial \Phi}{\partial t_{4}}= & \Delta\left[(\Delta \Phi)\left(\Delta\left(\Psi_{3} \Delta \Phi\right)-\Delta \Delta \Psi_{3}+2 \Psi_{3} \Delta \Delta \Phi\right)-\left(\Delta \Psi_{3}\right)(\Delta \Delta \Phi)\right. \\
& \left.-\Psi_{3} \Delta \Delta \Delta \Phi+\Delta\left(\Delta \Delta \Psi_{3}-\Delta\left(\Psi_{3} \Delta \Phi\right)-\Psi_{3} \Delta \Delta \Phi\right)\right]
\end{aligned}
$$

where $\Psi_{3}=\Delta((\Delta \Phi)(\Delta \Delta \Phi)-\Delta \Delta \Delta \Phi)$. Higher order equations can be worked out at will, but their structure cannot be easily described in closed form. Unfortunately, we have no systematic way to iterate the procedure and obtain $\Psi_{n}$ for all values of $n$. Likewise, the choice of supercontinual Lie algebras that enables to cast them into zero curvature will only be prescribed algorithmically.

Finally, one may formally append in the beginning of this list the heavenly equation, which is not parabolic but yields the Ricci flow by taking the square root of its time derivative operator.

\subsection{Integrability of higher order flows}

In order to understand how the hierarchy of higher order flows can be brought into zero curvature form, let us first consider the next example in the list given by the eighth order equation (8.6). In this case, the formal substitution $\partial / \partial t_{2}$ by $\partial / \partial \theta_{2}-\theta_{2} \partial / \partial t_{3}$ supplemented by the change of field variable $\Phi\left(z, \bar{z} ; t_{2}\right)$ to superfield $\mathcal{F}\left(z, \bar{z} ; t_{3}, \theta_{2}\right)$ can be directly applied to the continual Lie algebra for the Calabi flow. Using its description in terms of components $H_{i}(\varphi)$ and $X_{i}^{ \pm}(\varphi)$ with $i=0,1$, as given in section 5 , one obtains the following supercontinual Lie algebra with basic commutation relations

$$
\begin{aligned}
& {\left[H_{0}(\mathcal{F}), X_{0}^{ \pm}(\mathcal{G})\right]= \pm X_{0}^{ \pm}(\mathcal{F} \mathcal{G}), \quad\left[X_{1}^{ \pm}(\mathcal{F}), X_{0}^{\mp}(\mathcal{G})\right]= \pm H_{0}(\mathcal{F} \mathcal{G})} \\
& {\left[H_{0}(\mathcal{F}), X_{1}^{ \pm}(\mathcal{G})\right]= \pm X_{1}^{ \pm}(\mathcal{F} \mathcal{G}), \quad\left[X_{1}^{+}(\mathcal{F}), X_{1}^{-}(\mathcal{G})\right]=H_{1}(\mathcal{F} \mathcal{G})} \\
& {\left[H_{1}(\mathcal{F}), X_{1}^{ \pm}(\mathcal{G})\right]=\mp X_{0}^{ \pm}\left(\left(\mathcal{D}_{T} \mathcal{F}\right) \mathcal{G}\right)}
\end{aligned}
$$

whereas the rest are trivial,

$$
\begin{aligned}
& {\left[H_{1}(\mathcal{F}), X_{0}^{ \pm}(\mathcal{G})\right]=0, \quad\left[X_{0}^{+}(\mathcal{F}), X_{0}^{-}(\mathcal{G})\right]=0} \\
& {\left[H_{i}(\mathcal{F}), H_{j}(\mathcal{G})\right]=0}
\end{aligned}
$$

Here, to simplify notation, we drop the indices from the new time variable $t_{3}$ and the associated Grassmann variable $\theta_{2}$ and denote the corresponding super-evolution operator by $\mathcal{D}_{T}$, as before.

Then, starting from the zero curvature formulation of the Calabi flow written in terms of components and promoting the smearing functions to superfields, so that the gauge 
connections are taking values in the new supercontinual Lie algebra given above, we immediately arrive to the zero curvature formulation of equation (8.6). In particular, after some simplification of the original expressions used for the Calabi flow, we are led to propose the following choice of gauge connections,

$$
\begin{aligned}
& A_{+}=H_{0}(\partial \mathcal{F})+H_{1}(\partial \omega)+X_{1}^{+}(1), \\
& A_{-}=X_{0}^{-}(\partial \bar{\partial} \mathcal{F})+X_{1}^{-}\left(e^{\mathcal{F}}\right),
\end{aligned}
$$

where $\mathcal{F}$ and $\omega$ are now assumed to be $R^{1 \mid 1}$ mixed superfields with bosonic components that satisfy the relation

$$
\mathcal{D}_{T} \omega=-e^{-\mathcal{F}} \partial \bar{\partial} \mathcal{F}
$$

The zero curvature condition $F_{z \bar{z}}=\left[\partial+A_{+}, \bar{\partial}+A_{-}\right]=0$ becomes equivalent to the following equation for the superfield $\mathcal{F}(z, \bar{z} ; T)$

$$
\mathcal{D}_{T} e^{\mathcal{F}}=-\partial \bar{\partial}\left(e^{-\mathcal{F}} \partial \bar{\partial} \mathcal{F}\right)
$$

which when expanded in terms of components $\mathcal{F}=\Phi+\theta \Psi$ yields the system

$$
\partial_{t} e^{\Phi}=\partial \bar{\partial}(\Delta \Psi-\Psi \Delta \Phi), \quad \Psi=-\Delta \Delta \Phi .
$$

Eliminating $\Psi$ leads to the eighth order evolution equation (8.6), as advertised.

One can work out the form of the gauge connections, as well as the form of the supercontinual Lie algebra, in components by expanding everything in $R^{1 \mid 1}$ superspace. This will give rise to four bosonic components for each type of basic generators, which can be labeled as $H_{i j}(t)$ and $X_{i j}^{ \pm}(t)$ with $i$ and $j$ taking values 0 or 1 . The resulting expressions are quite lengthy and they are not included here. These generators, however, can be further promoted to elements of a supercontinual Lie algebra by changing $\partial / \partial t$ to $\mathcal{D}_{T}$ and using $R^{1 \mid 1}$ superfields as new smearing functions. This procedure can be repeated indefinitely, and, as a result, one obtains a recursive construction of an infinite hierarchy of continual Lie algebras that are associated to the hierarchy of higher order flows. Thus, any member of the family is cast into zero curvature form by the appropriate choice of algebra.

In general, a flow of order $2^{n+1}$, which is associated to a deformation variable $t$, is naturally obtained from a continual Lie algebra whose basic elements can be labeled as follows,

$$
H_{i_{1} i_{2} \cdots i_{n}}(t), \quad X_{i_{1} i_{2} \cdots i_{n}}^{ \pm}(t),
$$

with all indices taking values 0 or 1 . There are $2^{n}$ such independent elements for each type of generators, which follow by iterating the decomposition of $R^{1 \mid 1}$ superfields used at each level. Alternatively, they can be regarded as the bosonic components (in appropriate base) of three basic generators $H(T)$ and $X^{ \pm}(T)$, which are labeled by a supercontinuous index in $R^{1 \mid n}$ superspace with coordinates $T=\left(t ; \theta_{1}, \theta_{2}, \cdots, \theta_{n}\right)$, and likewise for their smearing functions. Thus, one may also consider the decomposition

$$
A(T)=A_{0}(t)+\sum_{i=1}^{n} \theta_{i} A_{i}(t)+\sum_{i<j} \theta_{i} \theta_{i} A_{i j}(t)+\cdots+\theta_{1} \theta_{2} \cdots \theta_{n} A_{12 \cdots n}(t)
$$


applied each basic generator $H(T)$ or $X^{ \pm}(T)$. The coefficients in the expansion are fully anti-symmetric in their indices, and, therefore, the number of independent components for each generator also sums up to $2^{n}$ in this parametrization.

It will be interesting to see whether the resulting hierarchy of infinite dimensional algebras can be put in more compact form in terms of $H(T), X^{ \pm}(T)$ so that the standard group theoretical methods of Toda field equations can be directly applicable to them for appropriate choice of Cartan operator. These steps will not be carried out explicitly here for the higher order equations but are left for the future.

\section{Generalization to arbitrary dimensions}

The hierarchy of geometric flows can be generalized to all Kähler manifolds of arbitrary dimension. Indeed, given the formal relation between the Ricci and Calabi flows, as outlined in all generality in section 2, we are led to propose a hierarchy of higher order geometric flows of the form

$$
\frac{\partial}{\partial t_{n}} g_{a \bar{b}}=\Omega_{a \bar{b}}^{(n)}(R)
$$

imposing the requirement $\partial_{t_{n}}^{2} g_{a \bar{b}}=-\partial_{t_{n+1}} g_{a \bar{b}}$ for all time variables $t_{n}$. Everything that was said before generalizes quite naturally to all dimensions, apart from the underlying algebraic structures that are only useful for the zero curvature formulation of the flows on Kähler manifolds of complex dimension 1 . We already have $\Omega_{a \bar{b}}^{(1)}=-R_{a \bar{b}}$ and $\Omega_{a \bar{b}}^{(2)}=$ $\partial_{a} \bar{\partial}_{b} R$, and therefore all higher order equations assume the general form

$$
\frac{\partial}{\partial t_{n}} g_{a \bar{b}}=\partial_{a} \bar{\partial}_{b} V_{n}
$$

for appropriately chosen expressions $V_{n}(R)$.

To illustrate the general structure of the equations, let us construct the next member of the hierarchy by computing the Calabi velocity of $R$, namely

$$
\frac{\partial}{\partial t_{2}} R=-\Delta \Delta R-R^{a \bar{b}} R_{a \bar{b}}
$$

It is convenient at this point to introduce the fourth order elliptic operator of Lichnérowicz, which is defined for each Kähler manifold as

$$
\mathcal{L} \varphi=D_{L} \varphi-g^{a \bar{b}} R_{, a} \varphi_{, \bar{b}}
$$

According to the definition of $D_{L}$ we have $\mathcal{L} R=\Delta \Delta R+R^{, a \bar{b}} R_{a \bar{b}}$, and, therefore, the next flow takes the form

$$
\frac{\partial}{\partial t_{3}} g_{a \bar{b}}=\partial_{a} \bar{\partial}_{b}(\mathcal{L} R)
$$

It is a parabolic equation with respect to the new time variable $t_{3}$ and provides an eight order non-linear differential equation for the components of the Kähler metric. Similarly, 
one may work out the form of higher order flows by iteration, but the final results are rather complicated. In general, the $n$-th flow is a parabolic equation of order $2^{n}$ with respect to the space coordinates.

In all cases, apart from the (unnormalized) Ricci flow, the deformations are volume preserving, i.e.,

$$
\frac{\partial}{\partial t_{n}} \int_{M} d V(g)=\int_{M} d V(g) \Delta V_{n}=0
$$

for $n \geq 2$. They also preserve the Kähler class of the metrics as for the Ricci and Calabi flows. In fact, for all these flows, the quadratic curvature functional $S(g)$ that was introduced in section 2 exhibits an absolute minimum value equal to $\left(\int_{M} R d V(g)\right)^{2} / \int_{M} d V(g)$ that is attained by constant curvature metrics, when they exist on the Kähler manifold $M$. Also, the extremal metrics on $M$ arise as local minima of $S(g)$ for all higher order flows as well.

More precisely, using the form of the flows $\partial_{t_{n}} g_{a \bar{b}}=\partial_{a} \bar{\partial}_{b} V_{n}(R)$, we first compute the variation of $S(g)$,

$$
\begin{aligned}
\partial_{t_{n}} S(g) & =\frac{\partial}{\partial t_{n}} \int_{M} R^{2}[g] d V(g)=-2 \int_{M} R\left(D_{L} V_{n}\right) d V(g) \\
& =-2\left(D_{L} V_{n}, R\right)=-2\left(V_{n}, D_{L} R\right)
\end{aligned}
$$

The result is obtained, as in section 2, by introducing the fourth order elliptic operator $D_{L}$ and removing the total derivative terms that arise in the course of the calculation. The Euler-Lagrange equation $D_{L} R=0$ describes the extremal values of $S(g)$ for all such flows, and the corresponding configurations are the extremal metrics on $M$, when they exist, satisfying the equivalent (but simpler) equation $L R=0$, as for the Calabi flow. Actually, all these extrema are local minima because one finds that the Hessian form of $S(g)$ in the directions $t_{n}$ and $t_{m}$ is

$$
\frac{\partial^{2} S(g)}{\partial t_{n} \partial t_{m}}=2\left(D_{L} \bar{D}_{L} V_{n}, V_{m}\right)
$$

when evaluated at the critical points. The operator $D_{L}$ commutes with its complex conjugate $\bar{D}_{L}$ when a critical metric is being used and $D_{L} \bar{D}_{L}$ is a strongly elliptic eighth order operator that is self-adjoint and positive semi-definite. Then, for any given flow, the second variation is manifestly positive semi-definite,

$$
\frac{\partial^{2} S(g)}{\partial t_{n}^{2}}=2\left(V_{n}, D_{L} \bar{D}_{L} V_{n}\right) \geq 0
$$

implying that all extrema are local minima, as required.

It is fair to say that the preceding analysis appeared for the first time in the original works of Calabi, $[6,7]$, who considered the general problem of deforming Kähler metrics on $M$ within a given cohomology class,

$$
\tilde{g}_{a \bar{b}}=g_{a \bar{b}}+\partial_{a} \bar{\partial}_{b} u
$$


using arbitrary $t$-dependent globally defined scalar functions $u(z, \bar{z})$. The hierarchy we have presented above corresponds to specific choices of $u$, as given by the potential functions $V_{n}\left(z, \bar{z} ; t_{n}\right)$. The ordinary Calabi flow corresponds to the choice $V_{2}\left(z, \bar{z} ; t_{2}\right)=R$ and has the special property that $S(g)$ decreases monotonically along it. All flows have critical points which are local (and possibly global) minima of $S(g)$ associated to extremal Kähler metrics. We see that the only directions of deformation of a critical metric in which the expression (9.9) vanishes are those tangent to the orbit formed by all holomorphic transformations of $M$ preserving the Kähler class of the metric. These transformations act as gauge group for the variational problem of minimizing the curvature functional $S(g)$ for they leave it invariant. Conversely, any flow that deforms the metric transversely to the gauge group orbit has strictly positive definite second derivative, as given by equation (9.9).

Finally, note that there are no curvature functionals in our disposal, at least for the moment being, which decrease monotonically along the corresponding higher order flows. It is an interesting variational problem to construct such expressions and study the long time behavior of their trajectories, as for the ordinary Calabi flow. Further implications of the higher order geometric deformations to problems of Kählerian geometry are left open for future work together with their possible physical applications.

\section{Conclusions and discussion}

We have provided a unifying framework for formulating geometric evolution equations driven by the intrinsic curvature on Kähler manifolds, so that the metric deforms to canonical form within a given cohomology class. In two dimensions, in particular, these equations admit zero curvature description with gauge connections taking values in appropriately chosen infinite dimensional Lie algebras that incorporate the deformation variable $t$ into their system. Such uneven treatment of the coordinates $(z, \bar{z})$ and $t$ allows to establish an algorithm for their formal integration in terms of a one-parameter family of two dimensional free fields, as in Toda field equations. At the same time, the evolution of any given initial metric exhibits dissipative behavior in $t$, since curvature perturbations around the canonical metric tend to decay exponentially fast. However, there is no contradiction of terms with the notion integrability in two dimensions, as $t$ becomes internal index of the algebra of gauge transformations in the corresponding zero curvature conditions. Thus, it is possible to bring the equations into two-dimensional integrable form and hide all dissipative behavior in $t$ in the internal space.

The notion of supercontinual Lie algebras that was introduced here for the first time, has been particularly useful for establishing the zero curvature formulation of the twodimensional Calabi flow by extending previously known results for the Ricci flow. This was implemented in practice by by extending $t$ to $R^{1 \mid 1}$ superspace with coordinates $(t, \theta)$ and promoting the time evolution operator to super-evolution. Further iteration of this procedure gave rise to an infinite hierarchy of higher order geometric flows, which exist 
on Kähler manifolds in all dimensions and they are integrable in two dimensions in the same sense that the Ricci and Calabi flows are. Actually, all these flows provide special examples of a more general variational problem posed by Calabi for deforming the metrics within a given Kähler class. In that context, one considers the quadratic curvature functional for a given Kähler manifold $M$ of arbitrary dimension, whose local minima define the notion of extremal metrics on $M$, such as constant curvature metrics, provided that there are no obstructions for their existence. The general class of flows introduced by Calabi, $\partial_{t} g_{a \bar{b}}=\partial_{a} \bar{\partial}_{b} u$, exhibit common qualitative behavior in that they deform any initial geometric configuration toward the extremal metrics. The standard Calabi flow corresponds to the deformation driven by the scalar curvature, $u=R$, but there can be more arbitrary choices of the $t$-dependent scalar function $u$. The hierarchy we have constructed here selects some special functions $u$ in a recursive way, such as $R$, $\mathcal{L} R$, etc, and the corresponding higher order flows were found to admit zero curvature formulation in two dimensions. It will be interesting to examine whether more general Calabi deformations can admit similar description in two dimensions using appropriately chosen infinite dimensional Lie algebras depending on the form of $u(z, \bar{z} ; t)$.

The infinite dimensional structures that arise in the algebraic description of twodimensional flows are quite novel, as they typically exhibit infinite growth. The prime example is the continual Lie algebra with Cartan operator $K=\partial / \partial t$ used for the Ricci flow, which grows exponentially fast beyond its local part. The supercontinual algebra with $K=\mathcal{D}_{T}$ that was used for the Calabi flow is also known to have infinite growth, and the same behavior is expected from the infinite dimensional algebras associated to all higher order flows. Luckily, the zero curvature formulation of these flows involves gauge connections that take values in the local part of the algebra, and, hence, the complete structure of the algebra is not required for their Toda-like description and the formal construction of their general solution. However, it is natural to expect that the explicit construction of two-dimensional integrals requires better handle on the commutation relations of the algebras used in each case, beyond their local part, and the existence of invariant forms. It is conceivable that there will be no algebraic integrals of these equations, as in some other examples of exactly solvable systems whose zero curvature formulation is based on infinite dimensional gauge groups, like the long-studied Halphen equations, [40]. These issues remain out of reach at the moment, as more mathematical work is required to bring the theory of such algebras, and their representations, to higher level of understanding. More generally, the very notion of integrability for twodimensional systems based on infinite dimensional gauge algebras calls for further work, as there are only some isolated examples in the literature so far; see, also, references [41] and [42] where other examples are analyzed and compared to more traditional systems whose integrability properties are compatible with the Painleve criterion.

The main physical application of the present work lies within the theory of spherical gravitational waves in vacuum in four space-time dimensions, as given by the general class of radiative Robinson-Trautman metrics. The formal integration of the RobinsonTrautman (two-dimensional Calabi) equation by group theoretical methods allows to 
express all type II solutions (in Petrov's classification) using two-dimensional free fields, as in all Toda field equations. However, there are hardly any explicit solutions known to this day that may depend on a finite number of moduli of the deformed two-dimensional sphere sitting inside the four-dimensional radiative metrics. If that were the case, some simple solutions of gravitational radiation in the exterior of bounded sources would have been possible to manufacture and study in exact terms. Put differently, there seems to be no reasonable mini-superspace description of the Robinson-Trautman-Calabi equation, unlike the simpler case of two-dimensional Ricci flows that admit consistent truncations and lead to interesting axially symmetric deformations of the round sphere, as the sausage model. Mini-superspace truncations of non-linear evolution equations, when they exist, are also tractable by more direct computational methods that do not rely on the Toda field theory interpretation of the equations and the free field parametrization of their general solution. Thus, it appears that exact models of gravitational radiation depend on an infinite number of parameters and there is only a formal power series expansion for them based on the group theoretical interpretation of the general solution. The class of type III Robinson-Trautman metrics was also shown to define an integrable two-dimensional system with gauge connections taking values in an infinite dimensional algebra identified with Kac's $K_{2}$ algebra of infinite growth.

Another interesting problem for future work is the generalization of the present formalism to include geometric evolution equations on supermanifolds. So far, there has been no systematic work in this direction and it certainly deserves more attention. A seemingly related problem is the supersymmetric generalization of the Ricci and Calabi flows on Kähler manifolds. It will be interesting to examine whether in two dimensions, in particular, there are appropriate choices of continual and supercontinual Lie superalgebras that can accommodate such generalizations in the form of zero super-curvature conditions. A different but illustrative example is provided by the $N=1$ supersymmetric generalization of the heavenly equation, whose bosonic version is viewed as continual Toda system in two dimensions with gauge connections taking values in the local part of the $S U(\infty)$ algebra. In the supersymmetric extension of the heavenly equation, [43], one considers the Lie superalgebra $\operatorname{sl}(N \mid N+1)$ in the large $N$ limit in which the system of simple roots is odd and corresponds to the super-principal embedding of $o s p(1 \mid 2)$ in it. As a result, the Cartan operator of the corresponding continual superalgebra is $\partial / \partial t$, equal to the square root of the Cartan operator of $S U(\infty)$, and it happens to coincide with the Cartan operator of the continual Lie algebra used for the Ricci flow. This occurrence might be only the beginning of a systematic pattern for constructing supersymmetric extensions of the hierarchy of higher order geometric flows, together with their zero curvature formulation, by also turning ordinary derivatives into supersymmetric covariant derivatives in two dimensions. It remains to be seen how far this construction can be pushed and what is the systematics behind it.

The use of infinite dimensional algebras that incorporate the time variable $t$ into their defining system of commutation relations might be of more general value for the reformulation of many dynamical problems other than the geometric evolution equations. If this 
possibility materializes it will certainly provide new ways to address difficult dynamical problems in terms of new classes of infinite dimensional Lie algebras and their representations. It may also fertilize the interplay between physics and mathematics in an area where Lie algebras of infinite growth become relevant and demand better understanding of the whole subject.

Finally, it is quite interesting to consider the interplay between physics and mathematics for other classes of geometric evolution equations and develop new tools for their study. In this context, a unified picture of intrinsic as well as extrinsic flows could emerge.

\section{Acknowledgements}

This work was supported in part by the European Research and Training Network "Constituents, Fundamental Forces and Symmetries of the Universe" under contract number MRTN-CT-2004-005104 and the INTAS program "Strings, Branes and Higher Spin Fields" under contract number 03-51-6346. I thank the Theory Division at CERN for hospitality and financial support during my sabbatical leave in the academic year

2004-05, where the main body of the present work was carried out in excellent and stimulating environment.

\section{Dedication}

This paper is dedicated to the glowing memory of an exceptional man and a great scientist, Bryce S. DeWitt. 


\section{References}

[1] H.-D. Cao, B. Chow, S.-C. Chu and S.-T. Yau eds, "Collected papers on Ricci flow", Series in Geometry and Topology, vol. 37, International Press, Somerville, 2003.

[2] B. Chow and D. Knopf, "The Ricci flow: An introduction", Mathematical Surveys and Monographs, vol. 110, American Mathematical Society, Providence, 2004.

[3] A.M. Polyakov, "Interaction of Goldstone particles in two dimensions. Applications to ferromagnets and massive Yang-Mills fields", Phys. Lett. B59 (1975) 79; "Gauge Fields and Strings", Contemporary Concepts in Physics, vol. 3, Harwood Academic Publishers, Chur, 1987.

[4] D. Friedan, "Nonlinear sigma models in $2+\epsilon$ dimensions", Phys. Rev. Lett. 45 (1980) 1057; "Nonlinear sigma models in $2+\epsilon$ dimensions", Ann. Phys. 163 (1985) 318.

[5] A. Tseytlin, "String vacuum backgrounds with covariantly constant null Killing vector and 2-d quantum gravity", Nucl. Phys. B390 (1993) 153.

[6] E. Calabi, "Extremal Kähler metrics", in Seminar on Differential Geometry, ed. S.-T. Yau, Annals of Mathematics Studies, vol. 102, Princeton University Press, 1982.

[7] E. Calabi, "Extremal Kähler metrics II", in Differential Geometry and Complex Analysis, ed. I. Chavel and H. Farkas, Springer-Verlag, Berlin, 1985.

[8] A. Futaki, "An obstruction to the existence of Einstein Kähler metrics", Invent. Math. $\underline{73}$ (1983) 437 .

[9] M. Levine, "A remark on extremal Kähler metrics", J. Diff. Geom. 21 (1985) 73.

[10] A. Futaki, "Kähler-Einstein Metrics and Integral Invariants", Lecture Notes in Mathematics, vol. 1314, Springer-Verlag, Berlin, 1988.

[11] G. Tian, "Canonical Metrics in Kähler Geometry", Lectures in Mathematics, Birkhäuser, Basel, 2000.

[12] P. Chrusciel, "Semi-global existence and convergence of solutions of the RobinsonTrautman (2-dimensional Calabi) equation", Commun. Math. Phys. 137 (1991) 289.

[13] X. Chen, "Calabi flow in Riemann surfaces revisited: a new point of view", Intern. Math. Res. Notices $\underline{6}$ (2001) 275; M. Struwe, "Curvature flows on surfaces", Ann. Sc. Norm. Super. Pisa Cl. Sci. (5) 1 (2002) 247.

[14] N. Manton, "Superevolution", J. Phys. A38 (2005) 6065.

[15] M. Saveliev, "Integro-differential non-linear equations and continual Lie algebras", Commun. Math. Phys. 121 (1989) 283. 
[16] M. Saveliev and A. Vershik, "Continual analogs of contragradient Lie algebras (Lie algebras with a Cartan operator and nonlinear dynamical systems), Commun. Math. Phys. 126 (1989) 367.

[17] A. Leznov and M. Saveliev, "Exactly and completely integrable nonlinear dynamical systems", Acta Appl. Math. 16 (1989) 1; "Group Theoretical Methods for Integration of Non-linear Dynamical Systems", Birkhäuser, Basel, 1992.

[18] C. Boyer and J. Finley, "Killing vectors in self-dual, Euclidean Einstein spaces", J. Math. Phys. 23 (1982) 1126; A. Das and J. Gegenberg, "Stationary Riemannian space-times with self-dual curvature", Gen. Rel. Grav. 16 (1984) 817.

[19] I. Bakas, "Area preserving diffeomorphisms and higher spin fields in two dimensions", in Supermembranes and Physics in $2+1$ Dimensions, ed. M. Duff, C. Pope and E. Sezgin, World Scientific, Singapore, 1990; Q.-H. Park, "Self-dual gravity as a large $N$ limit of the two-dimensional non-linear sigma model", Phys. Lett. B238 (1990) 287.

[20] I. Bakas and K. Sfetsos, "Toda fields of $S O(3)$ hyper-Kähler metrics and free field realizations", Int. J. Mod. Phys. A12 (1997) 2585.

[21] I. Bakas, "Renormalization group flows and continual Lie algebras", JHEP $\underline{0308}$ (2003) 013.

[22] I. Bakas, "Ricci flows and infinite dimensional algebras", Fortschr. Phys. $\underline{52}$ (2004) 464; "Ricci flows and their integrability in two dimensions", C. R. Physique $\underline{6}$ (2005) 175.

[23] V.A. Fateev, E. Onofri and Al.B. Zamolodchikov, "Integrable deformations of the O(3) sigma model. The sausage model", Nucl. Phys. B406 [FS] (1993) 521.

[24] I. Bakas, "On the integrability of spherical gravitational waves in vacuum", grqc/0504130.

[25] I. Robinson and A. Trautman, "Spherical gravitational waves", Phys. Rev. Lett. $\underline{4}$ (1960) 431; "Some spherical gravitational waves in general relativity", Proc. Roy. Soc. $\underline{\text { A265 }}$ (1962) 463.

[26] E.T. Newman, "Lienard-Wiechert fields and general relativity", J. Math. Phys. $\underline{15}$ (1974) 44.

[27] K.P. Tod, "Analogues of the past horizon in the Robinson-Trautman metrics", Class. Quant. Grav. $\underline{6}$ (1989) 1159.

[28] D. Kramer, H. Stephani, E. Herlt and M. MacCallum, "Exact Solutions of Einstein's Field Equations", Cambridge University Press, Cambridge, 1980. 
[29] C. Collinson and D. French, "Null tetrad approach to motions in empty space-time", J. Math. Phys. $\underline{8}$ (1967) 701.

[30] W. Natorf, "New vacuum Robinson-Trautman metrics", preprint gr-qc/0504056.

[31] J. Foster and E.T. Newman, "Note on the Robinson-Trautman solutions", J. Math. Phys. $\underline{8}$ (1967) 189.

[32] B. Lukacs, Z. Perjes, J. Porter and A. Sebestyen, "Lyapunov functional approach to radiative metrics", Gen. Relat. Grav. 16 (1984) 691.

[33] B. Schmidt, "Existence of solutions of the Robinson-Trautman equation and spatial infinity", Gen. Relat. Grav. 20 (1988) 65; A. Rendall, "Existence and asymptotic properties of global solutions of the Robinson-Trautman equation", Class. Quant. Grav. $\underline{5}$ (1988) 1339; D. Singleton, "On global existence and convergence of vacuum Robinson-Trautman solutions", Class. Quant. Grav. 7 (1990) 1333.

[34] P. Chrusciel, "On the global structure of Robinson-Trautman space-times", Proc.

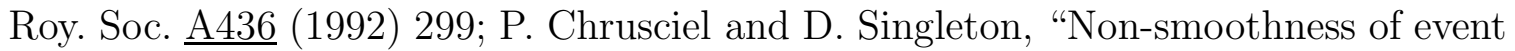
horizons of Robinson-Trautman black holes", Commun. Math. Phys. 147 (1992) 137.

[35] J. Bicak and J. Podolsky, "Cosmic no-hair conjecture and black-hole formation: An exact model with gravitational radiation", Phys. Rev. D52 (1995) 887; "Global structure of Robinson-Trautman radiative space-times with cosmological constant", Phys. Rev. D55 (1997) 1985.

[36] E. Glass and D. Robinson, "A nilpotent prolongation of the Robinson-Trautman equation", J. Math. Phys. $2 \underline{5}$ (1984) 3382.

[37] M. Vandyck, "On the time evolution of the Robinson-Trautman solutions", Class. Quant. Grav. 2 (1985) 77; "On the time evolution of some Robinson-Trautman solutions. II", Class. Quant. Grav. 4 (1987) 759.

[38] V.G. Kac, "Simple irreducible graded Lie algebras of finite growth", Math. USSR Izv. 2 (1968) 1271; "On simplicity of certain infinite dimensional Lie algebras", Bull. Amer. Math. Soc. 2 (1980) 311.

[39] J. Finley, "The Robinson-Trautman type III prolongation structure contains $K_{2}$ ", Commun. Math. Phys. 178 (1996) 375; "Estabrook-Wahlquist prolongations and infinite dimensional algebras", in Symmetry Methods in Physics, ed. A. Sissakian and G. Pogosyan, JINR Dubna, 1996.

[40] L. Takhtajan, "A simple example of modular forms as tau-functions for integrable equations", Theor. Math. Phys. 93 (1992) 1308; A. Maciejewski and J.-M. Strelcyn, "On the algebraic non-integrability of the Halphen system", Phys. Lett. A201 (1995) 161; S. Chakravarty and R. Halburd, "First integrals of a generalized DarbouxHalphen system", J. Math. Phys. 44 (2003) 1751. 
[41] R. Gebert, T. Inami and S. Mizoguchi, "The Painleve property, $W$-algebras and Toda field theories associated with hyperbolic Kac-Moody algebras", Int. J. Mod. Phys. A11 (1996) 5479.

[42] M. Ablowitz, S. Chakravarty and R. Halburd, "Integrable systems and reductions of the self-dual Yang-Mills equations", J. Math. Phys. $\underline{44}$ (2003) 3147.

[43] M. Saveliev and P. Sorba, "Solution of the Cauchy problem for a continuous limit of the Toda lattice and its super-extension", Lett. Math. Phys. 22 (1991) 119. 